\title{
UNIVERSITY OF HAWAI'I LIBRARY
}

\section{SUPPORT VECTOR MACHINES AND WAVELET PACKET ANALYSIS FOR FAULT DETECTION AND IDENTIFICATION}

\section{A THESIS SUBMITTED TO THE GRADUATE DIVISION OF THE UNIVERSITY OF HAWAI'I IN PARTIAL FULFILLMENT OF THE REQUIREMENTS FOR THE DEGREE OF}

\section{MASTER OF SCIENCE}

$\mathbf{I N}$

\section{ELECTRICAL ENGINEERING}

MAY 2006

\author{
By \\ Estefan M. Ortiz
}

Thesis Committee:

Vassilis L. Syrmos, Chairperson

Tep Dobry

Todd Reed 
We certify that we have read this thesis and that, in our opinion, it is satisfactory in scope and quality as a thesis for the degree of Master of Science in ELECTRICAL ENGINEERING.
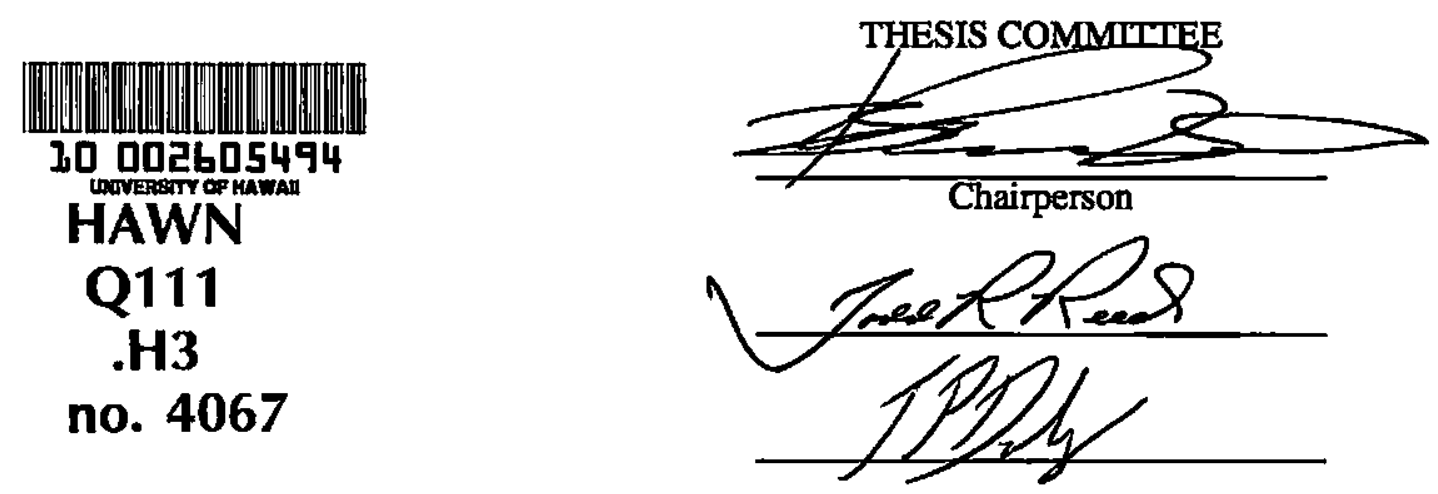


\section{Acknowledgements}

I am grateful to my advisor, Professor Vassilis L. Syrmos, for his enormous knowledge, patience, and support throughout my M.S. program at University of Hawai ' $i$. I would like to thank him for accepting me as his student. I also want to thank Professor Tep Dobry and Professor Tod Reed for their help in my thesis work. This work is dedicated to my family for their endless support in all endeavors to which I pursue. I would also like to thank my office mates, Ashish Babbar, Xudong Wang, and Mick West for their encouragement and support. 


\section{Contents}

Acknowledgements iii

List of Tables

List of Figures vii

$\begin{array}{ll}\text { Abstract } & \text { ix }\end{array}$

1 Introduction 1

2 Support Vector Machines 5

2.1 Support Vector Machines: Linearly Separable Patterns . . . . . . . . . 5

2.2 Support Vector Machines: Non-Separable Patterns . . . . . . . . . . . . 10

2.3 Nonlinear Support Vector Machines . . . . . . . . . . . . . . . 12

2.4 SVMs for Multi-Class Pattern Recognition . . . . . . . . . . . . . . . 15

2.4.1 One-Versus-All (Winner Takes All) . . . . . . . . . . 15

2.4.2 One-Versus-One Max votes wins . . . . . . . . . . . . 16

3 Wavelet Packet Analysis 18

3.1 Wavelet Analysis . . . . . . . . . . . . . . . . 18

3.2 Discrete Wavelet Transform . . . . . . . . . . . . . . 20

3.3 Wavelet Packet Analysis ................... 23

3.4 Feature Representation . . . . . . . . . . . . . . . 25

4 Fault Detection and Identification Scheme 31

4.1 System for FDI Application . . . . . . . . . . . . . . . 31

4.2 Fault Diagnosis Model . . . . . . . . . . . . . . . . . 32

5 Simulation Results 35

5.1 System description $\ldots \ldots \ldots \ldots \ldots \ldots \ldots \ldots$

5.1.1 Attitude-Control System of an Aircraft . . . . . . . . . 35

5.1.2 Sallen-Key Band-pass Filter . . . . . . . . . . . . . 37

5.2 FDI performance evaluation .................... 39 
5.2.1 Attitude-Control System of an Aircraft case . . . . . . . . . . 39

5.2.2 Sallen-Key Band-pass Filter case $\ldots \ldots \ldots$. . . . . . . 47

6 Conclusion 58

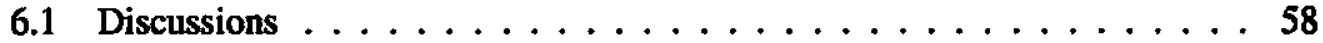

6.2 Future Work . . . . . . . . . . . . . . . . . . 59

Bibliography 


\section{List of Tables}

5.1 Attitude-Control System Component Values . . . . . . . . . . . . . . 37

5.2 Resistor and Capacitor Values for the Sallen-Key Bandpass Filter with center frequency of $25 \mathrm{kHz} \ldots \ldots \ldots$. . . . . . . . . . . . . 39

5.3 Component and Tolerance Values for DC Motor of the Attitude-Control System . . . . . . . . . . . . . . . . . . . . 39

5.4 Best Performance Breakdown: All Node Packets . . . . . . . . . . . . 44

5.5 Best Performance Breakdown: Best Tree Node Packets . . . . . . . . . . . 46

5.6 Comparison of the Two Feature Representation Methods Best Performance 47

5.7 Component Values and Tolerance Ranges for the Sallen-Key Bandpass Filter 48

5.8 Best Performance Breakdown: All Node Packets for the Sallen Key Bandpass Filter . . . . . . . . . . . . . . . . . . . 53

5.9 Best Performance Breakdown: Best Tree Node Packets for the Sallen Key Bandpass Filter . . . . . . . . . . . . . . . . . . 55

5.10 Comparison of the Two Feature Representation Methods Best Performance for the Sallen Key Bandpass Filter . . . . . . . . . . . . . . . 56 


\section{List of Figures}

1.1 Fault Diagnosis Model $\ldots \ldots \ldots \ldots$

2.1 Support Vector Machine two dimensional example . . . . . . . . . . 7

2.2 SVM: Non-separable case with slack variables . . . . . . . . . . . . . 12

2.3 Three class example of One-versus-All strategy . . . . . . . . . . 16

2.4 Three class example of One-versus-One strategy . . . . . . . . . . . 17

3.1 Third level wavelet decomposition of a time signal. $c_{j}(k), d_{j}(k)$ Approximation and detail coefficients at level $j$ respectively. . . . . . . . . 22

3.2 Daubechies wavelet of order $4 \ldots \ldots \ldots . \ldots . \ldots 23$

3.3 Third level Wavelet Packet decomposition of a time signal. . . . . . . . . 24

3.4 Wavelet Packet Feature Representation based on all Packets in Tree . . . . 27

3.5 Wavelet packet feature representation based on nodes from best basis tree . 29

4.1 System $\mathrm{T}$ decomposed into its respective subsystems . . . . . . . . . . 31

4.2 Example of a system's response to error . . . . . . . . . . . . . . 32

4.3 Block diagram of exciting a system in fault to collect training and testing data for FDI scheme. . . . . . . . . . . . . . . . . 33

4.4 Expanded Block Diagram of Proposed FDI Scheme . . . . . . . . . . . 34

5.1 Block diagram of the attitude-control system . . . . . . . . . . 35

5.2 Transfer Function Block diagram of the attitude-control system . . . . . . . 36

5.3 Expanded view of DC Motor's Transfer Function Block Diagram . . . . 36

5.4 Sallen-Key bandpass filter with center frequency of $25 \mathrm{kHz} \ldots \ldots$

5.5 Attitude-Control System FDI Generalization Error for One-verse-One Training with All Nodes for Features . . . . . . . . . . . . . . . . . . 42

5.6 Attitude-Control System FDI Generalization Error for One-verse-All Training with All Nodes for Features . . . . . . . . . . . . . . . . . 42

5.7 Attitude-Control System FDI Generalization Error. Best Curve Comparison of two Training Conventions with All Nodes as Features . . . . . . .43

5.8 Attitude-Control System FDI Generalization Error for One-verse-One Training with Best Basis Nodes as Features . . . . . . . . . . . . . . . 44

5.9 Attitude-Control System FDI Generalization Error for One-verse-All Training with Best Basis Nodes as Features . . . . . . . . . . . . . . 45 
5.10 Attitude-Control System FDI Generalization Error. Best Curve Comparison of two Training Conventions with Best Basis Nodes as Features . . . . 46

5.11 Sallen Key Bandpass FDI Generalization Error for One-verse-One Training with All Nodes for Features . . . . . . . . . . . . . . . . . . . . . . 51

5.12 Sallen Key Bandpass FDI Generalization Error for One-verse-All Training with All Nodes for Features . . . . . . . . . . . . . . . . . . . . . 51

5.13 Sallen-Key bandpass filter FDI Generalization Error. Best Curve Comparison of two Training Conventions with all node entropy measures as features 52

5.14 Sallen Key Bandpass FDI Generalization Error for One-verse-One Training with Best Basis Nodes for Features . . . . . . . . . . . . . . . 53

5.15 Sallen Key Bandpass FDI Generalization Error for One-verse-All Training with Best Basis Nodes for Features . . . . . . . . . . . . . . . . . 54

5.16 Sallen-Key bandpass filter FDI Generalization Error. Best Curve Comparison of two Training Conventions with all node entropy measures as features 55

5.17 Effect of noise on FDI scheme for the Sallen-Key bandpass filter . . . . . 57 


\begin{abstract}
This thesis examines a data driven fault detection and identification (FDI) method which uses Support Vector Machines (SVM) and the Wavelet Packet Transform (WPT). The primary focus of this thesis is to present a robust data driven fault diagnosis scheme. The investigated scheme has the capability to detect and identify faulty components of a given system through examination of its output due to a specified input. The use of the wavelet packet transform serves to draw out those features of the output response which best characterize each of the fault classes for the various components. Support vector machines are used as the diagnosis phase to detect and isolate faults of a given system.
\end{abstract}




\section{Chapter 1}

\section{Introduction}

Advanced engineering systems are becoming increasingly more complex. As a result, there has been a growing demand for reliable fault detection and identification (FDI) schemes. A fault is defined to be any deviation of the system outside its designed operation. Fault detection is the process of recognizing when a system has begun to operate outside its original design limits. Fault identification is the process of locating the most likely root cause of the detected failure within the system's components or subsystems. Fault detection and identification procedures generally fall into two broad categories; model based and data driven methods.

Model based FDI schemes are based on an accurate mathematical model of the system's dynamics as it relates to the individual subsystems. As an example, model based techniques often use of an observer-based residual generation to detect and isolate system faults. Roughly, this technique observes a set of defined variables $y_{i}$ from the actual system and compares them to the same variables $\hat{y}_{i}$ from the modeled nominal operation of the system. An error between the two observation is calculated as $e_{i}=y_{i}-\hat{y}_{i}$, and contains new information (or innovations) about the system. The error $e_{i}$ or a function of the error are used as residuals which carry the needed information about a possible fault in the system. Therefore, if a fault occurs the modeled nominal system will be unable to accurately track the actual system. Fault detection is then accomplished by examination of the magnitudes of the residuals [24]. In model based FDI the state observers are routinely designed as Kalman filters based on the mathematical model of the given system. Model based methods attempt to model various fault modes of the system by reformulating 
the initial mathematical model to reflect a variety of fault conditions. From the various fault models, a bank of state estimators is constructed each representing a predefined fault mode. Finally, the model based technique uses each of the modeled fault modes in combination with an advanced reasoning technique to determine if and where a fault has occurred $[24,29]$. Again, for a model based technique to effectively detect and isolate faults the underlying mathematical model of the process must realistically represent the system under consideration.

However, it is often the case that a mathematical model is unavailable or the current model does not accurately describe the complex system. It is in this context that data driven methods are developed. Data driven FDI methodologies rely on the assumption that the statistical characteristics of the observed data are relatively stationary across each of the fault and nominal classes. In general, data driven FDI methods attempt to construct a map between the observed features from the system's output and some decision space which corresponds to the fault and nominal operation classes. Formally, a data driven FDI scheme for a system can be defined as the triple $\left(C_{f}, F_{s}, \Theta\right)$ where:

1. $C_{f}$, is the set of possible fault classes or types of failures. This set will also include the nominal operation class of the system.

2. $F_{a}$, is the set of features or attributes associated to each of the fault classes given in $C_{f}$.

3. $\Theta$, is the fault diagnostic mapping where $F_{s}$ is the domain and $C_{f}$ is the range. $\Theta$ : $F_{s} \mapsto C_{f}$

Figure 1.1 shows a description of this general formulation for data driven FDI schemes. The observed system contributes to the formulation by providing the needed feature space $F_{s}$. However, it is not necessary that the feature space correspond to the raw observed outputs of the system. In fact, it is often more useful to transform the original output space of the system via some mapping function to a feature space which is able to effectively characterize and separate the various fault classes.

This thesis develops a data driven fault detection and isolation method which employs support vector machines as the diagnosis machine $\Theta$ and a feature extraction phase which consists of the wavelet packet transform and a feature representation method based 


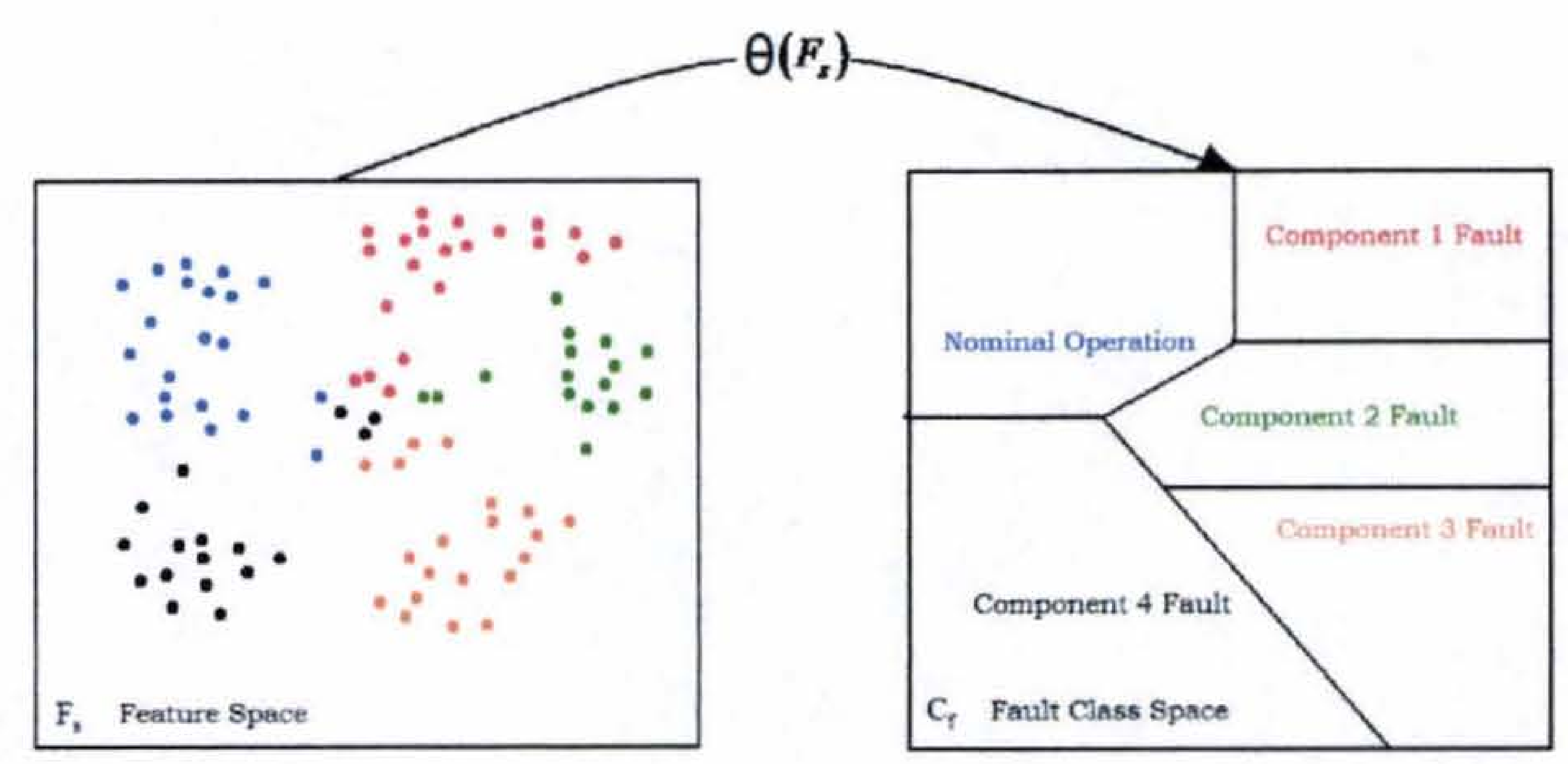

Figure 1.1: Fault Diagnosis Model

an entropy metric defined over the wavelet packets. The primary use of a feature extraction phase is to effectively transform the output space of a system to a feature space that better characterizes the possible fault classes. This is especially important when training a machine to recognize patterns associated to the various fault classes.

Support vector machines were principally introduced by Vapnik [26] and in recent years have been implemented successfully to many practical problems $[15,20]$. A support vector machine is a binary hyperplane classifier rooted in statistical learning theory and is an approximate implementation of the method of structural risk minimization. Hyperplane classifiers attempt to construct a dividing hyperplane between two classes. Under the formulation of SVMs a optimal hyperplane is one which is able to correctly classify the training samples and achieve the largest possible margin between each of the classes. Support vector machines are well suited for fault diagnosis because they are able to achieve desirable generalization properties without incorporating problem-domain knowledge [17]. Also, their generalization performance is independent of the dimensionality of the input samples [3]. This is particulary useful in fault diagnosis problems because the number of attributes chosen to be the base of diagnostics is unlimited. This thesis develops SVMs as the fault diagnosis machine. In particular, SVMs are trained on samples obtained from the various faulty and nominal operation modes. For which it is important the samples adequately characterize the salient features of each of the fault classes. 
In recent applications, wavelet based signal analysis has shown to provide an effective means of feature extraction from time varying signals [30, 22, 14]. In general, wavelet based feature extraction provides a method to view signals in a time-scale representation, where the scale is related to frequency content of the signal. However, the wavelet transform is not well suited to characterize signals exhibiting high frequency components. To overcome this deficiency a generalization of the wavelet transform was developed principally by Coifman and Wickerhauser in [10]. The wavelet packet transform offers a method to extract features found in the higher frequencies. In addition it also allows for a wider representation of a signal across a rich library of redundant wavelet bases. It is this property which allows for a better time-scale resolution and provide an FDI scheme with well defined features across each fault class.

To demonstrate the performance of the proposed fault diagnosis technique two systems are studied. The first system is an attitude-control system of an aircraft which is developed in [21]. This system is readily found on most modern aircraft and is primarily driven by a DC motor. Consequently, the proposed FDI scheme focuses on detecting and isolating faults occurring in the DC motor. The second system examined is a Sallen-Key bandpass filter. In this system the proposed FDI scheme is developed to diagnose faults arising from errors within the components of the filter. In this example, the proposed FDI scheme is designed to detect and identify faults in the resistors and capacitors of the filter. In both examples the effectiveness of the proposed data driven FDI technique is examined in detail.

The outline of the rest of this thesis is as follows. In Chapter 2 support vector machines and their extension to the multi-class pattern recognition problems is developed. In Chapter 3 the preprocessing phase based on the wavelet packet transform and feature representation through an entropy measure defined on the wavelet packets is derived. Chapter 4 spells out the proposed FDI method and the type of systems for it is applicable. In Chapter 5 the FDI scheme is applied to two different systems 1.Attitude-Control System of an Aircraft and 2. Sallen-Key Band-pass Filter. Finally discussions and future work are given in Chapter 6. 


\section{Chapter 2}

\section{Support Vector Machines}

This chapter describes the formulation of support vector machines. In the first two sections SVMs are developed under assumption that the training samples are linearly separable and then extended to the cases where the training set is not separable. The next section describes how SVMs are used when the decision surface is not a linear combination of the training samples. Nonlinear implementation of an SVM is accomplished through a kernel function which allows for ease of implementation in computing the necessary inner products in the nonlinear space. This thesis describes and uses the Gaussian kernel function for the nonlinear implementation of SVMs. The final section explains how SVMs are extended to the multi-class pattern recognition problems. Two of the more popular multi-class extensions, One-verses-All and One-verses-One are developed and examined in this thesis.

\subsection{Support Vector Machines: Linearly Separable Pat- terns}

The goal of a hyperplane classifier is to construct a decision surface between two classes such that the generalization error is minimized. Formally, given a set of feature vectors $\mathrm{x}_{i} \in \Re^{d}$ and their corresponding class labels $y_{i} \in\{+1,-1\}$ the training set is formed as the indexed pair $\left\{\left(x_{i}, y_{i}\right)\right\}_{i=1}^{N}$. Where feature vectors $x_{i}$ with labels +1 belong to Class 1 and those feature vectors $x_{i}$ with labels -1 belong to Class 2 . In the linearly 
separable case there exists a deciding hyperplane which is able to separate the training data. This separating hyperplane will have the following form,

$$
f(\mathbf{x})=\mathbf{w} \cdot \mathbf{x}+b
$$

Where $\mathbf{w} \in \Re^{d}$ and is the normal vector to the separating hyperplane and $b \in \Re$ is the scalar bias. Therefore, if $\mathbf{x}$ belongs to Class $1(+1$ label) then $f(\mathbf{x}) \geq 0$ and conversely if $\mathbf{x}$ belongs to Class $2\left(-1\right.$ label) decision function will be negative, $f(x)<0$. Let $d_{+}$and $d_{-}$ denote the shortest distances from the positive and negative points respectively. For a given weight vector $\mathrm{w}$ and bias $b$ the margin of separation $\rho$ is defined as the separation between the hyperplane and the closest feature vectors from each class and is expressed as $\rho=$ $d_{+}+d_{-}$. It has been shown that a good hyperplane classifier is one which is able to correctly classify the training samples while at the same time creating the widest margin of separation $[26,24]$. Roughly, when the margin of separation is maximized the generalization error on unseen test data tends to its minimum value. This argument is rooted in statistical learning theory and is described in detail in $[24,26,17,7]$. Support vector machines are the practical implementation of the maximum margin classifier concept. In particular a SVM formulates the problem of finding the separating hyperplane which maximizes the margin of separation $\rho$ as a quadratic programming problem for which there are computationally optimal algorithms. As an example of the following formulation Figure. 2.1 shows the separation of two classes in two dimensions.

The optimal hyperplane is achieved by formulating the problem as a quadratic optimization problem. In the linearly separable case $w$ and $b$ must satisfy the following constraints for the given training samples $\left\{\left(\mathbf{x}_{i}, y_{i}\right)\right\}_{i=1}^{N}$,

$$
\begin{gathered}
\mathbf{w} \cdot \mathbf{x}_{1}+b \geq 1 \quad \text { for } y_{i}=1 \\
\mathbf{w} \cdot \mathbf{x}_{1}+b \leq-1 \quad \text { for } y_{i}=-1 .
\end{gathered}
$$

Where the above constraints can be combined to form one set of inequalities,

$$
y_{i}\left(\mathbf{w} \cdot \mathbf{x}_{i}+b\right)-1 \geq 0 \quad \forall i
$$




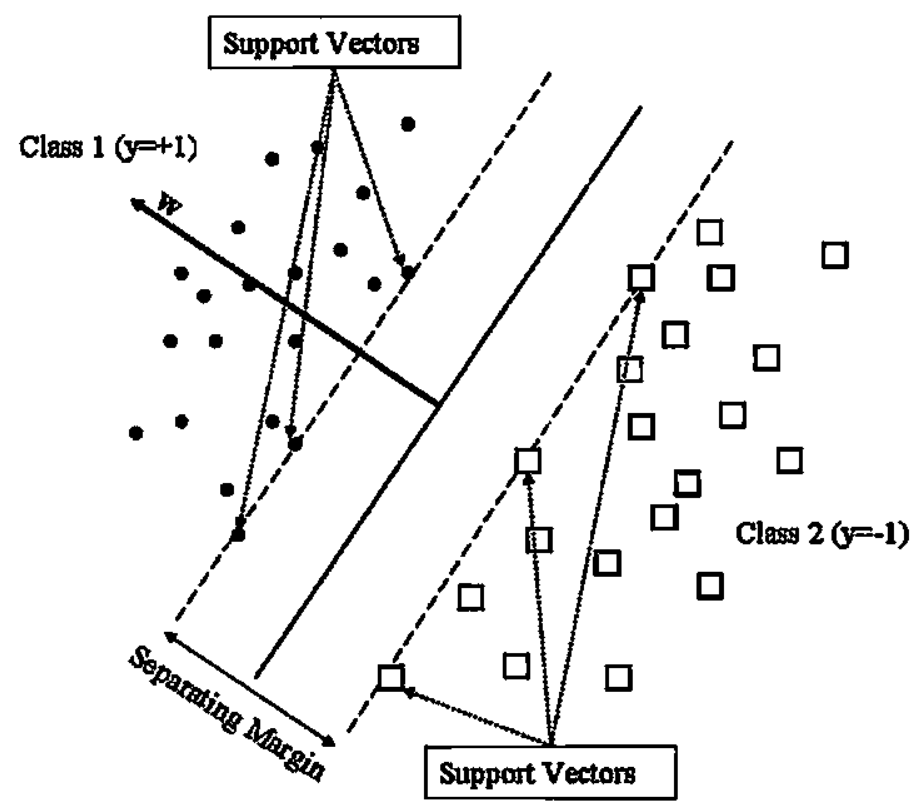

Figure 2.1: Support Vector Machine two dimensional example

The points for which the equality in (2.1.2) hold, lie on the hyperplane $H_{1}$ given as,

$$
H_{1}: \quad \mathbf{w} \cdot \mathbf{x}_{i}+b=1
$$

For which, the distance $\left(d_{+}\right)$to the deciding hyperplane $f(\mathbf{x})$ is $1 /\|\mathbf{w}\|$. Similarly, those points in which the equality in (2.1.2) hold lie on the hyperplane $\mathrm{H}_{2}$ defined as,

$$
H_{2}: \quad \mathbf{w} \cdot \mathbf{x}_{i}+b=-1
$$

which has a distance $\left(d_{-}\right)$to the deciding hyperplane $f(\mathbf{x})$ as $1 /\|\mathbf{w}\|$. The points for which the equalities in (2.1.2) and (2.1.3) hold are known as the support vectors and are the points which lie the closest to the separating hyperplane $f(\mathrm{x})$. The margin of separation is thus defined in terms of the distances from $f(\mathbf{x})$ to the support vectors from each class given as,

$$
\begin{aligned}
\rho & =d_{+}+d_{-} \\
& =\frac{1}{\|\mathbf{w}\|}+\frac{1}{\|\mathbf{w}\|} \\
& =\frac{2}{\|\mathbf{w}\|}
\end{aligned}
$$


Consequently, addition or removal of a support vector directly affects the decision surface. From (2.1.7 it is seen that the problem of maximizing the margin of separation $\rho$ is equivalent to minimizing the Euclidean norm of the weight vector $\mathbf{w}$.

The following formulation of support vector machines follows the derivations found in $[7,17]$. Given training samples $\left\{\left(x_{i}, y_{i}\right)\right\}_{i=1}^{N}$, the problem is to find the optimum hyperplane subject to the constraints given in equation (2.1.4) and which minimizes the following cost function,

$$
\Phi(\mathbf{w})=\frac{1}{2}\|\mathbf{w}\|^{2}
$$

This formulation is known as the primal problem and is characterized by its convex cost function $\Phi(w)$ and linear constraints found in (2.1.4). The method of Largrange multipliers is used to solve this constrained optimization problem [4]. The Lagrangian function is formed as,

$$
L(\mathbf{w}, b, \alpha)=\frac{1}{2}\|\mathbf{w}\|^{2}-\sum_{i=1}^{N} \alpha_{i}\left[y_{i}\left(\mathbf{w} \cdot \mathbf{x}_{i}+b\right)-1\right] .
$$

where $\alpha_{i}$ are the Lagrange multipliers. Therefore, in the primal formulation $L(w, b, \alpha)$ must be minimized with respect to $\mathrm{w}$ and $b$ and at the same time require the derivatives of $L(w, b, \alpha)$ with respect to $\alpha_{i}$ vanish subject to the constraint that $\alpha_{i} \geq 0$. The primal problem is a convex quadratic programming problem because its objective function is convex, and those points which satisfy the constraints also form a convex set. This implies that the equivalent dual problem can be formed and solved instead $[4,7]$. The solution to the dual problem is determined by maximizing $L(w, b, \alpha)$ with respect to $\alpha_{i}$ such that the derivatives of $L(w, b, \alpha)$ with respect to $\mathrm{w}$ and $b$ must vanish, all subject to the constraints of $\alpha_{i} \geq 0$. The requirement that the derivatives of $L(w, b, \alpha)$ with respect to $\mathbf{w}$ and $b$ vanish yields the following conditions,

$$
\begin{aligned}
& \frac{\partial L(\mathbf{w}, b, \alpha)}{\partial \mathbf{w}}=\mathbf{0} \\
& \frac{\partial L(\mathbf{w}, b, \alpha)}{\partial b}=0
\end{aligned}
$$

Solving for $w$ gives, 


$$
\mathbf{w}=\sum_{i=1}^{N} \alpha_{i} y_{i} \mathbf{x}_{i}
$$

and from (2.1.11),

$$
\sum_{i=1}^{N} \alpha_{i} y_{i}=\mathbf{0}
$$

Note the solution for $w$ is found as an expansion of the training samples and is unique because of the convexity of the cost function found in (2.1.8). However, unlike $w$ the coefficients $\alpha_{i}$ are not required to be unique. From the Karush-Kuhn-Tucker conditions of optimization theory [4] the coefficients $\alpha_{i}$ which are non-zero at the saddle point yield the following conditions,

$$
\alpha_{i}\left[y_{i}(\mathbf{w} \cdot \mathbf{x}+b)-1\right]=0
$$

Therefore, the support vectors are those training samples $\mathbf{x}_{i}$ for which $\alpha_{i}>0$ and lie on one of the hyperplanes defined in (2.1.5),(2.1.6). The dual problem is formed by expanding $L(w, b, \alpha)$ and substituting the equality constraints found in (2.1.12), and (2.1.13). This forms the following objective function,

$$
W(\alpha)=\sum_{i=1}^{N} \alpha_{i}-\frac{1}{2} \sum_{i=1}^{N} \sum_{j=1}^{N} \alpha_{i} \alpha_{j} y_{i} y_{j} \mathbf{x}_{i} \cdot \mathbf{x}_{j}
$$

where

$$
\alpha_{i} \geq 0 \quad i=1,2, \ldots N .
$$

The dual problem can be stated as, given the training set $\left\{\left(\mathbf{x}_{i}, y_{i}\right)\right\}_{i=1}^{N}$ find the Lagrange multipliers $\left\{\alpha_{i}\right\}_{i=1}^{N}$ such that the objective function $W(\alpha)$ is maximized subject to the constraints of (2.1.13),(2.1.16). The final decision surface is thus found as, 


$$
\begin{aligned}
f(\mathbf{x}) & =\mathbf{w} \cdot \mathbf{x}+b \\
& =\left(\sum_{i=1}^{N} \alpha_{i} y_{i} \mathbf{x}_{i}\right) \cdot \mathbf{x}+b \\
& =\sum_{i=1}^{N} \alpha_{i} y_{i} \mathbf{x}_{i} \cdot \mathbf{x}+b
\end{aligned}
$$

Equation (2.1.14) is used to determine the bias $b$. In particular, $b$ is found by selecting any training points for which the corresponding $\alpha$ are nonzero and solving (2.1.14) in terms of $b$. For a numerically stable solution for $b$ it is wise to average $b$ over all such training samples.

It is important to note that the problem is defined entirely in terms of the training samples. Furthermore, the training samples appear in the objective function $W(\alpha)$ and in the final solution as inner products. The fact that the training samples appear as inner products play an important role in extending SVMs to nonlinear pattern classification problems. The above formulation assumed that the two classes were linearly separable. This is an ideal condition but one not readily found in practical problems. The next section develops SVMs for the circumstances patterns are not separable.

\subsection{Support Vector Machines: Non-Separable Patterns}

To extend the formulation of SVMs to the situations where classes are not linearly separable a non-negative slack variable $\zeta$ is introduced into the two original constraints found in Eqns. (2.1.2) and (2.1.3),

$$
\begin{gathered}
\mathbf{w} \cdot \mathbf{x}_{1}+b \geq 1-\zeta_{i} \quad \text { for } y_{i}=1 \\
\mathbf{w} \cdot \mathbf{x}_{1}+b \leq-1+\zeta_{i} \text { for } y_{i}=-1 .
\end{gathered}
$$

The relaxed constraints formed in (2.2.1) and (2.2.2) can be combined to form one set of inequalities given as, 


$$
y_{i}\left(\mathbf{w} \cdot \mathbf{x}_{i}+b\right) \geq 1-\zeta_{i} \quad \text { for } i=1 \ldots N .
$$

For an error to occur the respective slack variable $\zeta$ must exceed unity. The introduced slack variables $\zeta_{i}$ "measure the deviation of a data point from the ideal condition of pattern separability." [17] The data points which fall within the margin of separation and on the correct side of the separating boundary will have associated slack variables $0 \leq \zeta_{i} \leq 1$. The data points which do not fall on the correct side of the margin will have associated slack variables $\zeta_{i}>1$. Figure 2.2 shows a two-dimensional example of the non separable case. In this formulation the support vectors are those points which satisfy Eqn. 2.2.3 and which $\zeta_{i}>0$. It follows that to assign an extra cost for errors the original objective function found in Eqn. (2.1.8) is reformulated as,

$$
\Phi(\mathbf{w}, \zeta)=\frac{1}{2}\|\mathbf{w}\|^{2}+C \sum_{i=1}^{N} \zeta_{i}
$$

where $C$ is referred to as the regularization parameter and dictates the tradeoff between the complexity of the SVM and the number of nonseparable points [17]. The larger the regularization parameter $C$ is the higher the penalty there is for errors. In this thesis $C$ is determined experimentally and its effect on the generalization error is studied for each of the considered systems. Following the procedure to find $w$ and $b$ in the linearly separable case the Wolf dual problem is to maximize $W(\alpha)$ found as,

$$
W(\alpha)=\sum_{i=1}^{N} \alpha_{i}-\frac{1}{2} \sum_{i=1}^{N} \sum_{j=1}^{N} \alpha_{i} \alpha_{j} y_{i} y_{j} \mathbf{x}_{i} \cdot \mathbf{x}_{j}
$$

subject to the following constraints.

$$
\begin{gathered}
0 \leq \alpha_{i} \leq C \quad i=1 \ldots N . \\
\sum_{i=1}^{N} \alpha_{i} y_{i}=0
\end{gathered}
$$

The solution for $w$ is again calculated as.

$$
\mathbf{w}=\sum_{i=1}^{N_{S}} \alpha_{i} y_{i} \mathbf{x}_{i}
$$


where $N_{S}$ is the number of support vectors. Note that the only difference between the formulation of the linearly separable case from the non-separable case is found in the constraints on the Lagrange multipliers $\alpha_{i}$ Eqn.2.2.6. In the separable case $\alpha_{i}$ need only be nonnegative. Whereas, in the non-separable case $\alpha_{i}$ needs to be nonnegative and bounded from above by the determined regularization parameter $C$.

The power of support vector machines lie in their ability to transform a non separable problem in the original input space to a possibly linear separable problem in some higher dimensional feature space. As mentioned before the the fact that the training samples appear in the solution as inner products will allow for this effective extension via kemel functions.

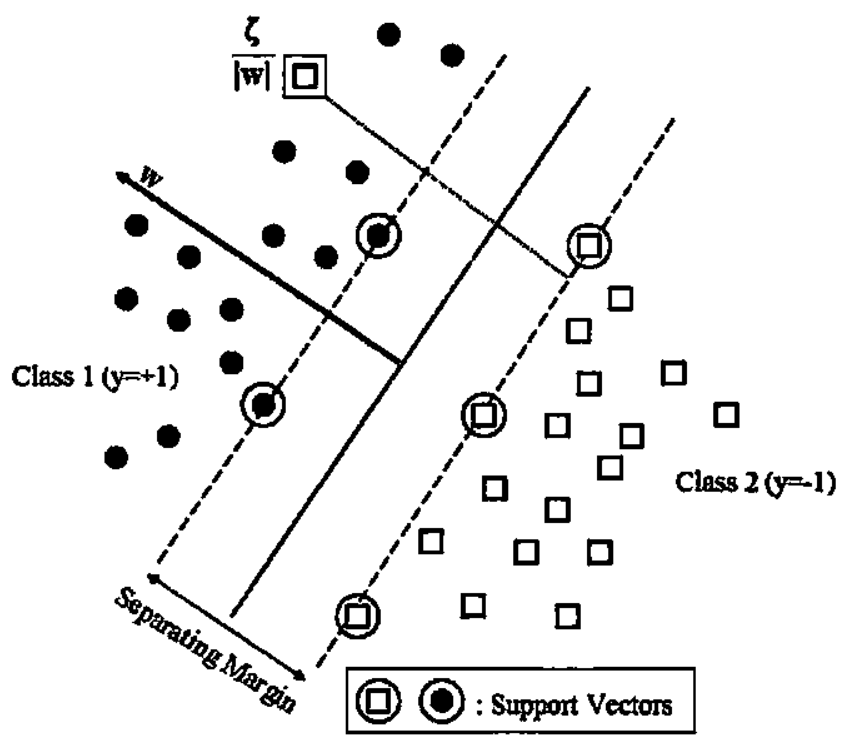

Figure 2.2: SVM: Non-separable case with slack variables

\subsection{Nonlinear Support Vector Machines}

Thus far the formulation of support vector machines has considered only linear hyperplanes as decision surfaces. This formulation can be extended to the cases where the decision surface is nonlinear. This is accomplished by first mapping the training data to 
higher possibly infinite dimensional feature space. The SVM algorithm is then computed in this feature to produce the desired nonlinear decision surface.

Formally, the training patterns $\left\{\mathrm{x}_{1} \ldots \mathrm{x}_{N}\right\}$ exist in some input space $\Re^{d}$ and a nonlinear mapping function $\varphi(\mathbf{x})$ exists such that it projects the training samples from $\Re^{d}$ to some higher dimensional space $H$ given as,

$$
\varphi: \Re^{d} \rightarrow H .
$$

Since the training samples appear in the SVM algorithm as inner products in the Wolf dual formulation Eqn.(2.2.5) and in the computation of $w$ Eqn. (2.2.8) the algorithm will only change by replacement of $\mathrm{x}$ with its map $\varphi(\mathrm{x})$ in Eqns. (2.2.5) and (2.2.8). These changes are reflected in the following,

$$
\begin{gathered}
W(\alpha)=\sum_{i=1}^{N} \alpha_{i}-\frac{1}{2} \sum_{i=1}^{N} \sum_{j=1}^{N} \alpha_{i} \alpha_{j} y_{i} y_{j} \varphi\left(\mathbf{x}_{i}\right) \cdot \varphi\left(\mathbf{x}_{j}\right) \\
\mathbf{w}=\sum_{i=1}^{N} \alpha_{i} y_{i} \varphi\left(\mathbf{x}_{i}\right) .
\end{gathered}
$$

This projection allows for class separation in the feature space $H$ when it cannot be clearly separated in the original input space. The SVM algorithm then constructs the deciding hyperplane $f(\mathbf{x})$ in the feature space $H$. The inner product $\varphi(\mathbf{x}) \cdot \varphi(\mathbf{y})$ in the higher dimensional feature space can be replaced by a suitable kernel function $K\left(\mathbf{x}_{i}, \mathbf{x}_{j}\right)$ given as,

$$
K\left(\mathbf{x}_{i}, \mathbf{x}_{j}\right)=\varphi\left(\mathbf{x}_{i}\right) \cdot \varphi\left(\mathbf{x}_{j}\right)
$$

The kernel function for an inner product in a higher dimensional feature space allows the SVM algorithm to compute the inner products without using the nonlinear map $\varphi(x)$ directly. This is particulary useful when the nonlinear map $\varphi(\mathbf{x})$ is difficult or impossible to compute directly. In terms of the nonlinear map $\varphi(\mathbf{x})$ the decision surface $f(\mathbf{x})$ is formulated as, 


$$
\begin{aligned}
f(\mathbf{x}) & =\mathbf{w} \cdot \varphi(\mathbf{x})+b \\
& =\left(\sum_{i=1}^{N_{S}} \alpha_{i} y_{i} \varphi\left(\mathbf{x}_{i}\right)\right) \cdot \varphi(\mathbf{x})+b \\
& =\sum_{i=1}^{N_{S}} \alpha_{i} y_{i} \varphi\left(\mathbf{x}_{i}\right) \cdot \varphi(\mathbf{x})+b
\end{aligned}
$$

For which the inner product found in the last line of the (2.3.5) is replaced with its corresponding kernel function. This substitution yields the following decision surface in terms of the appropriate kernel function $K\left(\mathbf{x}_{i}, \mathbf{x}_{j}\right)$,

$$
f(\mathbf{x})=\sum_{i=1}^{N} \alpha_{i} y_{i} K\left(\mathbf{x}_{i}, \mathbf{x}\right)+b
$$

Where the computation of $w$ to $H$ via $\varphi(\cdot)$ is avoided through the use of the kemel function $K\left(\mathbf{x}_{i}, \mathbf{x}_{j}\right)$. Admissible kemel functions in which to extend SVMs to the nonlinear domain are those which satisfy Mercer's condition [7]. Mercer's condition states that there exits a kernel expansion, $K(\mathbf{x}, \mathbf{y})$ for a mapping function $\varphi(\mathbf{x})$ if and only if for any $g(\mathbf{x})$ such that,

$$
\int g(\mathbf{x})^{2} d \mathbf{x}<\infty
$$

then

$$
\int K(\mathbf{x}, \mathbf{y}) g(\mathbf{x}) g(\mathbf{y}) d \mathbf{x} d y \geq 0
$$

Two of the more commonly used kernel functions are the polynomial kernel function and the Gaussian radial basis function which are respectively,

$$
\begin{gathered}
K\left(\mathbf{x}_{i}, \mathbf{x}_{j}\right)=\left(a \mathbf{x}_{i}^{T} \mathbf{x}_{j}+c\right)^{p} \\
K\left(\mathbf{x}_{i}, \mathbf{x}_{j}\right)=e^{-\gamma\left\|\mathbf{x}_{i}-\mathbf{x}_{j}\right\|^{2}}
\end{gathered}
$$

As mentioned earlier the dimension of the feature space could be infinite making direct computation of the nonlinear map difficult. The kernel function found in (2.3.10) gives an 
example of a feature space having infinite dimensionality whose inner product is computed by the defined kernel function. This thesis uses the Gaussian kernel function found in (2.3.10) to extend SVMs to the nonlinear domain. In particular, this thesis examines the effect $\gamma$ from equation (2.3.10) has on the generalization performance of the proposed FDI scheme. The best value found experimentally is used for a detailed performance analysis of the developed FDI scheme. As stated before SVMs are a binary classifier. The following section develops two methods which are used in this thesis to extend SVMs to the multiclass pattern recognition problems.

\subsection{SVMs for Multi-Class Pattern Recognition}

Support vector machines are binary classifiers and research to extend their use to the $M$-class pattern recognition problems is still ongoing. However, several effective methods have been developed and implemented to a variety of problems. The $M$-class pattern recognition problem is to build a decision function based on training examples sampled from each of the known classes. Formally, a decision function $f(\mathbf{x})$ is constructed from the training vectors $x_{i}$ and corresponding labels $\omega_{i}$ given as

$$
\left\{\left(\mathbf{x}_{i}, \omega_{i}\right)\right\}_{i=1}^{N}=\left\{\left(\mathbf{x}_{1}, \omega_{1}\right),\left(\mathbf{x}_{2}, \omega_{2}\right) \ldots\left(\mathbf{x}_{N}, \omega_{N}\right)\right\}
$$

Where $\mathbf{x}_{i} \in \Re^{d}$ and $\omega_{i} \in\{1, \ldots, M\}$.

Two of the more popular extensions of support vector machines to the multi-class problems are one-versus-all and one-versus-one. The one-versus-all method uses a winner take all strategy while the one-versus-one method uses a max-wins voting strategy each of the these methods are discussed in detail below. This thesis makes use of both one-versusall and one-versus-one for training and testing the developed FDI scheme.

\subsubsection{One-Versus-All (Winner Takes All)}

The One-Versus-All method is the simplest strategy to extend support vector machines to the $M$-class pattern recognition problems. This strategy builds $M$ binary SVM classifiers, one for each of the $M$ classes. The $i_{t h}$ classifier $f_{C_{t}}(\mathbf{x})$ is trained to identify the 
$\omega_{i}$ class from all the remaining classes. In training, the data examples associated to the concerned class $\omega_{i}$ are assigned positive labels and all remaining data examples are assigned negative labels. The classifiers $f_{C_{t}}(\mathbf{x})$ built for each of the respective classes are combined to form a final decision function $f_{C}(\mathbf{x})$. This final decision function is given as,

$$
f_{\mathbf{C}}(\mathbf{x})=\arg \max _{i}\left\{f_{C_{i}}(\mathbf{x})\right\}_{i=1}^{M}
$$

Therefore, a given test sample $\mathbf{x}$ is presented to each of the classifiers and the final label is determined by the classifier whose evaluation for $\mathbf{x}$ is the largest. Figure 2.3. shows an example of this process in a three class situation.

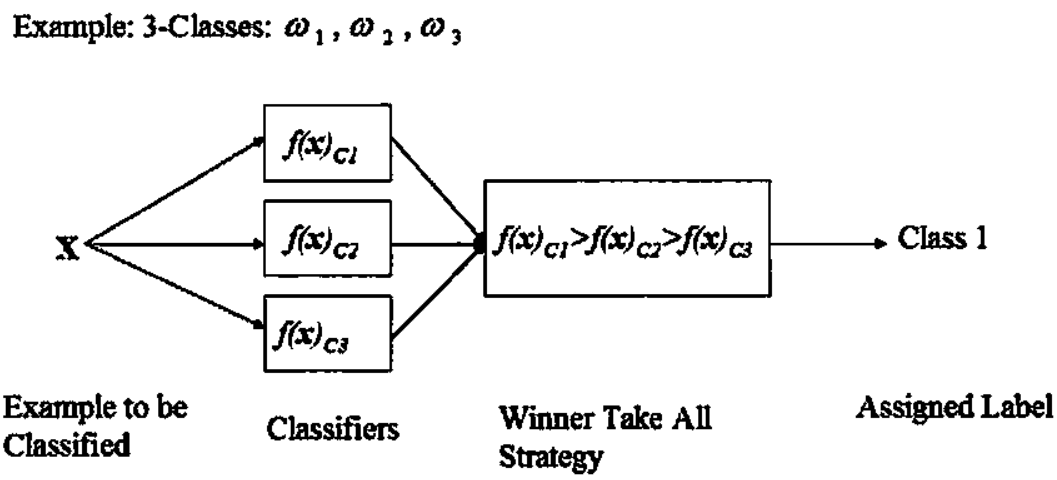

Figure 2.3: Three class example of One-versus-All strategy

In terms of fault diagnosis this method can be used to reject certain decisions or possibilities of a fault. In other words, a threshold $\theta$ can be used as a measure of confidence such that any decision $f_{C_{t}}(\mathrm{x})$ which falls below the threshold is discarded. In terms of fault diagnosis this is a means to present an ordered list of the most likely root causes of failure. Where the list is formed from those decisions which meet the threshold criteria. The main disadvantage of this approach is that it is heuristic. The outputs of binary classifiers trained for each class may not be on the same scale.

\subsubsection{One-Versus-One Max votes wins}

The One-Versus-One strategy builds a binary classifier for each pair of distinct classes. For an $M$ class problem this method constructs $\frac{M(M-1)}{2}$ binary classifiers. Let 
$f_{C_{i j}}(\mathbf{x})$ represent the binary classifier trained on examples from classes $\omega_{i}$ and $\omega_{j}$ where class $\omega_{i}$ is assigned the positive label and $\omega_{j}$ is assigned negative label. Once all classifiers have been trained the final label assigned to a unseen example $\mathbf{x}$ is done by a maximum voting strategy. In other words, each classifier assigns a label to $x$ and the label with the majority of votes is assigned as the final class label for $\mathbf{x}$. Formally, let $D_{j}$ represent the number of SVMs whose decision is the $\omega_{j}$ class,

$$
D_{j}=\left|\left\{k \mid f_{C_{i, j}}(\mathbf{x})=\omega_{j}\right\}\right|
$$

The final decision function is formed as,

$$
f_{\mathbf{C}}(\mathbf{x})=\arg \max _{j} D_{j}
$$

Figure 2.4. shows an example of this process in the three class situation.

Example: 3-Classes: $\omega_{1}, \omega_{2}, \omega_{3}$

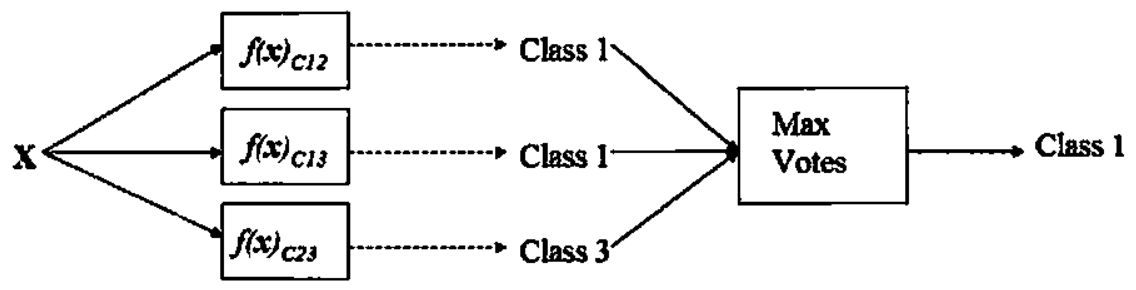

$\begin{array}{llll}\begin{array}{l}\text { Example to be } \\ \text { Classified }\end{array} \quad \text { Classifiers } & \begin{array}{l}\text { Determined } \\ \text { Classifier Label }\end{array} & \text { Voting Strategy Assigned Label }\end{array}$

Figure 2.4: Three class example of One-versus-One strategy

If a tie should occur, say between class $\omega_{i}$ and $\omega_{j}$ the tie is broken by taking the decision of the classifier $f_{C_{i j}}(\mathbf{x})$ trained to distinguish between the respective classes. In terms of fault diagnosis this method offers the ability to list the most probable cause of an error in terms of the votes a respective fault class receives. Even though the final decision takes the max vote to be the final label. When implemented for use it is often more beneficial to give the maintainer a list of probable causes in descending order of votes per class (possible fault locations). However, this feature comes at the cost of solving $\frac{M(M-1)}{2}$ quadratic programming problems. Each of the described methods are evaluated in this thesis. 


\section{Chapter 3}

\section{Wavelet Packet Analysis}

This chapter explains the preprocessing phase of the fault detection and identification scheme. The preprocessing phase consists of the Wavelet Packet Transform (WPT) and an entropy metric used to map the wavelet packet coefficents to a feature vector representation. The wavelet packet transform is a generalization of the discrete wavelet transform (DWT), and it is instructive to develop the WPT from the DWT. Therefore, the first two sections of this chapter develop wavelet analysis and its implementation to sampled signals via the DWT. The next section describes the WPT as a generalization of the DWT. The final section details the two methods of feature representation based on entropy measures of the wavelet packets.

\subsection{Wavelet Analysis}

The wavelet transform (WT) analyzes a time signal $f(t)$ at multiple levels of resolution. When the wavelet transform is used on a time signal what results is a twodimensional, time-scale representation of $f(t)$. More specifically, the wavelet transform measures the time-frequency of the spectral components at different time-frequency resolutions [23]. To decompose a time signal $f(t)$ an analyzing function $\psi(t)$ and the set $\left\{\psi_{a, b}(t)\right\}$ which consists of shifted and scaled versions of $\psi(t)$ is constructed as,

$$
\psi_{a, b}(t)=\frac{1}{\sqrt{a}} \psi\left(\frac{t-b}{a}\right), \quad a>0, \quad a, b \in \Re .
$$

where $\psi(t)$ is commonly referred to as the mother wavelet. For an appropriately chosen $\psi(t)$, the set $\left\{\psi_{a, b}(t)\right\}$ will form an orthonormal basis for $L^{2}(\Re)$. Where $L^{2}(\Re)$ is the space 
of square integrable functions. The parameter $b$ controls the translations of $\psi(t)$ and $a$ is the scale parameter which determines the dilations and contractions of the mother wavelet. Therefore, the continuous wavelet transform (CWT) is defined as,

$$
C(a, b)=\frac{1}{\sqrt{a}} \int_{-\infty}^{\infty} f(t) \psi\left(\frac{t-b}{a}\right) d t .
$$

Where the CWT decomposes a function $f(t) \in L^{2}(\Re)$ into its wavelet coefficients $C(a, b)$. For completeness the original signal can be reconstructed from the inverse transform,

$$
f(t)=\frac{1}{C_{\psi}} \int_{-\infty}^{\infty} \int_{-\infty}^{\infty} C(a, b) \psi\left(\frac{t-b}{a}\right) d b \frac{d a}{|a|^{2}}
$$

where $C_{\psi}$ is given as,

$$
C_{\psi}=\int_{-\infty}^{\infty} \frac{|\Psi(\zeta)|^{2}}{|\zeta|} d \zeta
$$

and $\Psi(\zeta)$ is the Fourier transform of $\psi(t) . C_{\psi}$ is known as the admissibility constant and for the inverse transform to exist $C_{\psi}$ must satisfy the admissibility condition that is, $C_{\psi}<+\infty$

The coefficients found in Eqn.(3.1.2) measure how correlated $f(t)$ is with the mother wavelet at time $b$ and scale $a$. Therefore the coefficients $C(a, b)$ will also measure the frequency content of $f(t)$ at time $b$. Thus, the wavelet transform offers a method to draw out time-frequency information in the form of the coefficients $C(a, b)$. To examine this further the Fourier transform of $\psi(t)$ is taken and given as,

$$
\frac{1}{\sqrt{a}} \psi\left(\frac{t}{a}\right) \Leftrightarrow \sqrt{a} \Psi(a \Omega)
$$

Accordingly, when mother wavelet $\psi(t)$ is dilated $(a>1)$ it exhibits low frequency characteristics. Conversely, when $\psi(t)$ is contracted $(a<1)$ it exhibits high frequency characteristics. To use the WT in a discrete form the DWT is developed in following section. 


\subsection{Discrete Wavelet Transform}

The DWT is the continuous wavelet transform applied to sampled time signals and has its formulation and implementation rooted in multiresolution analysis (MRA) [23, 12]. The DWT of a given time signal splits the resolution levels in terms of octave bands. Such that each level of decomposition corresponds to a frequency range half as wide as the level above it and twice as wide as the level below it [9]. In particular, given a mother wavelet $\psi(t)$ the dyadic set of translations and dilations are used to form the set of wavelet functions $\left\{\psi_{j, k}(t)\right\}$ given as,

$$
\psi_{j, k}(t)=2^{\frac{k}{2}} \psi\left(2^{j} t-k\right) \quad j, k \in \mathrm{Z}
$$

For an appropriately chosen $\psi(t)$, the set $\left\{\psi_{j, k}(t)\right\}$ will also form an orthonormal basis for $L^{2}(\Re)$. Allowing $f(t) \in L^{2}(\Re)$ to be expressed as an expansion of the basis functions $\left\{\psi_{j, k}(t)\right\}$.

$$
\begin{aligned}
f(t) & =\sum_{j, k}\left\langle f(t), \psi_{j, k}(t)\right\rangle \psi_{j, k}(t) \\
& =\sum_{j, k} \lambda_{j}(k) \psi_{j, k}
\end{aligned}
$$

Where \langle\rangle is the inner product defined on $L^{2}(\Re)$. The set of coefficients $\lambda_{j}(k)$ are called the discrete wavelet transform of $f(t)$.

As a consequence of multiresolution analysis a family of scaling functions can be formed as,

$$
\phi_{j, k}(t)=2^{\frac{j}{2}} \phi\left(2^{j} t-k\right) \quad j, k \in \mathrm{Z}
$$

such that $\phi_{j, k}$ and $\psi_{j, l}$ are orthogonal. This leads to an expansion of $f(t)$ in terms of the scaling function and the mother wavelet given as,

$$
f(t)=\sum_{k} c_{M}(k) \phi_{M, k}(t)+\sum_{j=1}^{M} \sum_{k} d_{j}(k) \psi_{j, k}(t)
$$


where $M$ is the desired level of decomposition. The coefficients $c_{M}(k)$ and $d_{j}(k)$ are commonly denoted as the approximation and detail coefficients respectively. The approximation and detail coefficients are calculated by inner products,

$$
\begin{aligned}
& c_{j}(k)=\left\langle f(t), \phi_{j, k}\right\rangle=\int f(t) \phi_{j, k}(t) d t \\
& d_{j}(k)=\left\langle f(t), \psi_{j, k}\right\rangle=\int f(t) \psi_{j, k}(t) d t
\end{aligned}
$$

To implement the DWT the multiresolution formulation gives the following two-scale relation,

$$
\psi(t)=\sqrt{2} \sum_{k} g(k) \phi(2 t-k)
$$

Where $\phi(t)$ can be written as a recursive relationship,

$$
\phi(t)=\sqrt{2} \sum_{k} h(k) \phi(2 t-k)
$$

From (3.2.7) the mother wavelet $\psi(t)$ is constructed from the defined scaling function $\phi(t)$. And as a consequence of (3.2.7) and (3.2.8) the coefficients $c_{j}(k)$ and $d_{j}(k)$ can be found recursively as,

$$
\begin{aligned}
& c_{j}(k)=\sum_{m} h(m-2 k) c_{j+1, m} \\
& d_{j}(k)=\sum_{m} g(m-2 k) d_{j+1, m}
\end{aligned}
$$

The coefficients $c_{j}$ and $d_{j}$ are often referred to as the approximation and detail coefficients respectively. Equations (3.2.7) and (3.2.8) can be solved such that the sequences $h(k)$ and $g(k)$ will have only finite nonzero coefficients and allows for efficient algorithms to compute the wavelet coefficients (approximations and details) [12]. Implementation of DWT is achieved through a procedure developed by Mallat which consists of quadrature mirror filters (QMFs) for subband coding [23]. The implementation of the DWT based on QMFs consists of a low pass filter $H$ and a high pass filter $G$. In which the frequency responses of $H$ and $G$ are mirror images of each other. In general, $h(k)$ are the coefficients of the low 
pass filter $H$ and $g(k)$ are the coefficients of the high pass filter $G$. To compute the wavelet coefficients, $f(t)$ is first convolved with the respective filters. Then the approximation coefficients are again filtered using the respective filters. The algorithm then proceeds in the same manner taking those coefficients from the low-pass filter branch and again filtering them into the approximation and detail coefficients at the next level of decomposition. Figure 3.1 shows how this implementation is performed. This implementation of the DWT via filters is known as the fast wavelet transform (FWT). The FWT also makes use of a process called dyadic decimation in which the number of points in the coefficients is decreased by a factor of two at each level of decomposition. In other words, if the signal has $2^{n}$ points then the number of coefficients to represent the signal at the next level of decomposition will be $2^{n-1}$.

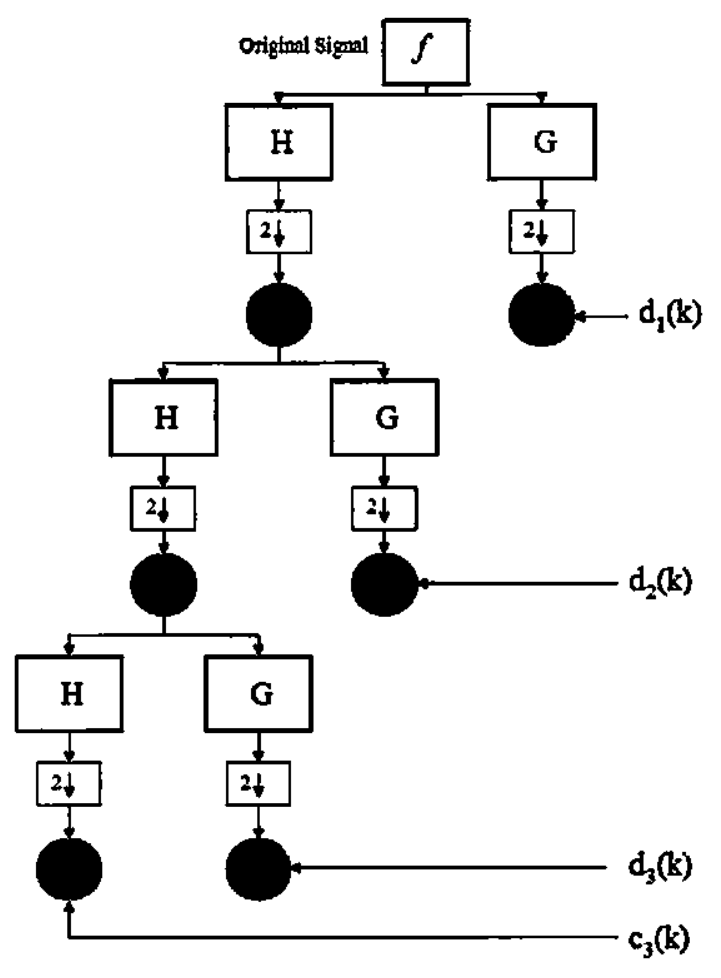

Figure 3.1: Third level wavelet decomposition of a time signal. $c_{j}(k), d_{j}(k)$ Approximation and detail coefficients at level $j$ respectively.

This thesis uses the orthogonal Daubechies Wavelet D4 transform as the mother wavelet. This mother wavelet was chosen based on experimental results which will be pre- 
sented in Chapter 5. This type of wavelet is characterized by having the maximal number of vanishing moments for the given support. Vanishing moments of a wavelet refer to its ability to represent polynomial behavior or information in a signal. In addition, the Daubechies wavelets have been widely used in solving problems such as, finding self-similarity properties of a signal or fractal patterns, detection of signal discontinuities, etc. Figure 3.2 displays the scaling and wavelet functions for the D4 transform.
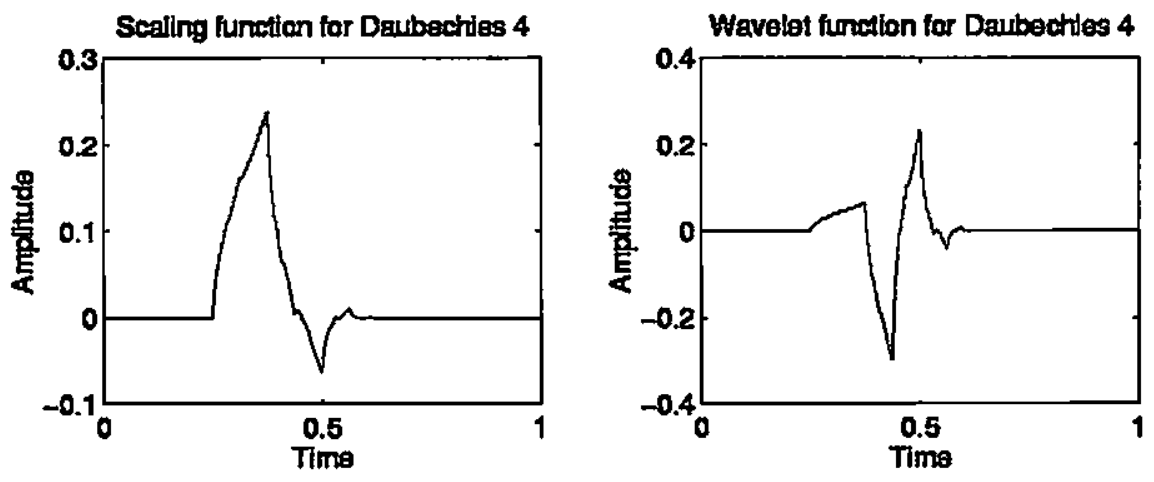

Figure 3.2: Daubechies wavelet of order 4

\subsection{Wavelet Packet Analysis}

The wavelet packet transform is a generalization of the wavelet transform which provides a rich family of orthonormal bases in which to decompose a time signal. The wavelet transform is simply one member of the bases constructed from the WPT. As previously described, the wavelet transform can provide good localization in both the time and frequency domains. However, discrimination of frequency is sacrificed in order to achieve good time localization at higher levels of decomposition. In other words, the DWT provides us with a constant- $Q$ filtering which in turn allows for the low frequencies to have narrow bandwidths and the high frequencies to have wide bandwidths [11]. It is therefore difficult to distinguish the features that contain high frequency elements using features based on the wavelet coefficients from the DWT. To resolve this problem Coifman and Wickerhauser [10] developed the wavelet packet transform which provides for a better resolution at the 
higher frequency end of the spectrum. In fact, the wavelet packet transform provides for a greater flexibility. Unlike the WT the WPT allows for the analysis to focus on any part of the time-frequency domain. Since the wavelet packet transform is a generalization of the DWT it offers a larger set of features to be used for pattern recognition. Recall from Fig. 3.1 the DWT takes the signal $f(t)$ and passes it through the scaling and wavelet filters, low-pass and high-pass filters respectively. After filtering and decimation there will be two types of coefficients, those from the high-pass filter (details) and those from the lowpass filter (approximations). The algorithm then proceeds in the same manner taking those coefficients from the low-pass filter branch and again filters them into the approximation and detail coefficients at the next level of decomposition. In contrast, the wavelet packet transform splits both the lowpass and highpass bands at all stages of decomposition. This analysis forms the full binary decomposition tree as seen in Fig. 3.3.

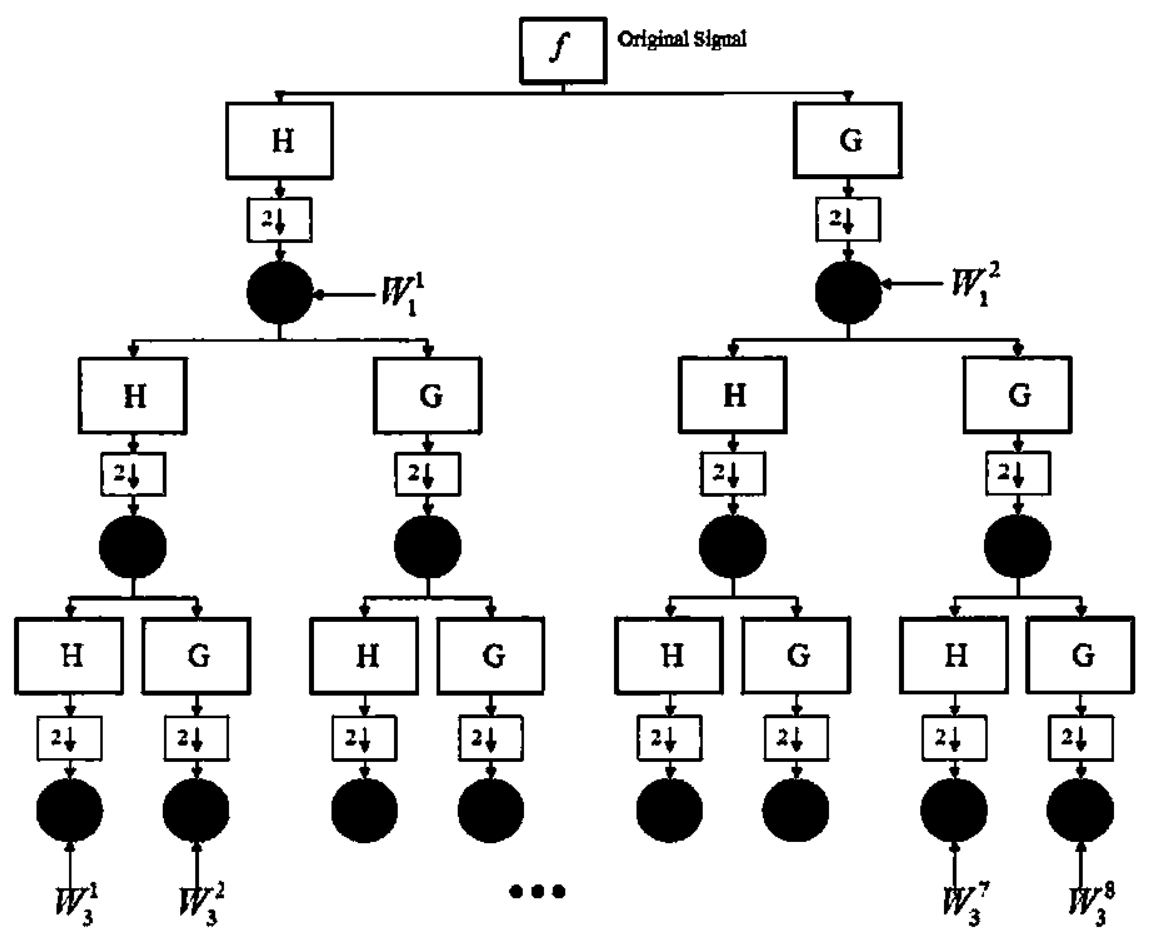

Figure 3.3: Third level Wavelet Packet decomposition of a time signal.

Formally, a wavelet packet function $W_{j, k}^{n}(t)$ is defined as , 


$$
W_{j, k}^{n}(t)=2^{j / 2} W^{n}\left(2^{j} t-k\right)
$$

where the integers $j$ and $k$ are the scale and translation variables. The variable $n$ is denoted as oscillation parameter. The wavelet packet functions are then formed by the following recursive relationships,

$$
\begin{aligned}
W_{0,0}^{2 n}(t) & =\sqrt{2} \sum_{k} h(k) W_{1, k}^{n}(2 t-k) \\
W_{0,0}^{2 n+1}(t) & =\sqrt{2} \sum_{k} g(k) W_{1, k}^{n}(2 t-k) .
\end{aligned}
$$

Where, $W_{0,0}^{0}(t)=\varphi(t)$ and $W_{0,0}^{1}(t)=\psi(t)$ are the scaling and mother wavelet functions respectively [30]. Again, the sequences $h(k)$ and $g(k)$ are in general the coefficients of the low-pass and high-pass filters of the QMFs. To determine the wavelet packet coefficients, $w_{j}^{n}(k)$ the inner product $\left\langle f(t), W_{3, k}^{n}\right\rangle$ is computed as,

$$
\begin{aligned}
w_{j}^{n}(k) & =\left\langle f(t), W_{j, k}^{n}\right\rangle \\
& =\int f(t) W_{j, k}^{n}(t) d t
\end{aligned}
$$

As mentioned before the full wavelet packet decomposition can be computed by successive implementation of the QMFs not only on the approximation coefficients but also on the detail coefficients down to a specified level of decomposition. The described decomposition method results in a wavelet packet tree seen in Fig. 3.3.

\subsection{Feature Representation}

Direct use of the wavelet packet coefficients for pattern recognition for fault detection and identification is difficult and the overall performance and accuracy tends to be poor. One of the main reasons for the poor performance is due to the lack of the translationinvariant property of the wavelet packet transform. This difficulty arises when there is a shift in time in signals which are otherwise the same. In which direct use of the coefficients will not well represent the same signal consistently when there is a translation in time. This 
is problematic for pattern recognition purposes because the very features needed to distinguish between each of the classes are inconsistent due to a simple shift in time. However, a information measure can be defined on the wavelet packets such that it will provide a more robust feature representation. This measure should be able to quantify the information at each band of frequency at the $j_{t h}$ level of decomposition for each of the wavelet packet nodes. This thesis explores the use of the Shannon entropy measure defined on the wavelet packet sequences expanded by the WPT. More specifically, features for a given signal are constructed from such a measure. This thesis examines two types of feature representation:

1. Feature representation defined by the entropy measure applied to all the wavelet packet nodes

2. Feature representation defined by the entropy measure applied to those nodes resulting from the best basis algorithm developed by Coifman and Wickerhauser [10].

Building feature vectors in terms of an entropy measure will better establish signatures which are able to discriminate between each of the fault classes of system.

The concept of a cost function or an energy function over the sequences of wavelet packet was first described in [10]. The measure should describe the concentration, or number of coefficients needed to accurately describe the sequence. Formally, for a coefficient sequence $W_{j}^{n}=w_{j}^{n}(k)$ the map $E$ from the sequence $w_{j}^{n}(k)$ to the set of reals $\Re, E: w_{j}^{n}(k) \mapsto \Re$ is defined such that it satisfies the following two conditions,

$$
\begin{gathered}
E(0)=0 \\
E\left(W_{j}^{n}\right)=\sum_{k} E\left(w_{j}^{n}(k)\right) .
\end{gathered}
$$

A function $E$ which satisfies the above two conditions is referred to as an additive information cost function. Thus for a signal $f(t)$ decomposed into to its full wavelet packet tree the cost function $E$ defined for the $n_{t h}$ node at the $j_{t h}$ level, $W_{j}^{n}$ is defined as,

$$
E\left(W_{j}^{n}\right)=\sum_{k} E\left(w_{j}^{n}(k)\right)
$$

This allows for a natural feature vector representation for $f(t)$ through its WPT decomposition. More specifically, the feature vector $\mathrm{E}$ is given as, 


$$
\mathbf{E}=\left[E_{1,1}, E_{1,2}, E_{2,1} \ldots E_{j, n}\right]
$$

where,

$$
\begin{aligned}
E_{j, n} & =E\left(W_{j}^{n}\right) \\
& =\sum_{k} E\left(w_{j}^{n}(k)\right)
\end{aligned}
$$

Which is the entropy function computed for all nodes of the full wavelet packet decomposition tree. The ordering of the wavelet packet node indices is from top to bottom and read from left to right. The ordering of the wavelet packet nodes in constructing the feature vector $\mathbf{E}$ is arbitrary and will not have an impact on the support vector machine in training and testing so as long the ordering is uniform across all signals used to build the training and testing sets. Figure 3.4 depicts this process of feature vector construction for a third level wavelet packet decomposition tree.

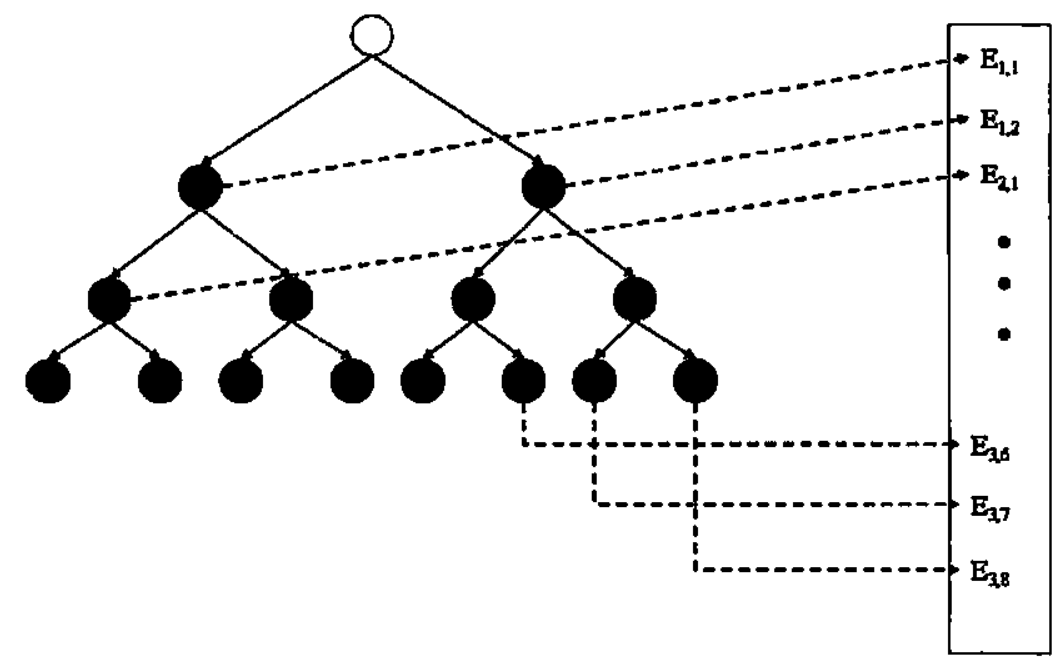

Figure 3.4: Wavelet Packet Feature Representation based on all Packets in Tree

This thesis uses the Shannon entropy function as the additive information cost function. This cost function is used because it is the measure used by Coifman and Wickerhauser in their best basis algorithm, which will be described later in detail. 
The Shannon entropy function defined on a wavelet packet sequence is given as,

$$
E\left(W_{j}^{n}\right)=-\sum_{k}\left(w_{j}^{n}(k)\right)^{2} \log \left(w_{j}^{n}(k)\right)^{2}
$$

where $\left(w_{j}^{n}(k)\right)^{2} \log \left(w_{j}^{n}(k)\right)^{2}$ is taken to be 0 if $w_{j}^{n}(k)=0$

The entropy function $E\left(W_{j}^{n}\right)$ measures the amount of energy in the coefficient sequence. In other words, if $E\left(W_{j}^{n}\right)$ is large the coefficients in the sequence are all about the same and $E\left(W_{j}^{n}\right)$ is small if all but a few coefficients are negligible. The second feature representation constructs a feature vector from those nodes found from the best basis algorithm developed by Coifman and Wickerhauser.

The algorithm developed by Coifman and Wickerhauser provides a method to search the wavelet packet tree to find the "best basis"in which to represent a signal adaptively [10]. This algorithm was originally used to as a method to efficiently compress a variety of signals. The goal was to find the orthonormal basis which has the lowest information cost. Formally, let $B_{n, j}$ be the basis of vectors of the $n$th node at the $j$ th level of the wavelet packet tree. Define $A_{n, j}$ as the best basis for the decomposed signal restricted to the span $B_{n, j}$. Thus, for a given signal $x \in \Re^{d}, B x$ denotes the vector of coefficients of $x$ in the orthonormal basis $B$ and similarly $E(B x)$ denotes the information cost of $x$ in the basis $B$. The best basis algorithm given the cost function $E(\cdot)$ defined in (3.4.6) can be found recursively as

$$
A_{n, j}=\left\{\begin{array}{lr}
B_{n, j} & \text { if } E\left(B_{n, j} x\right) \leq E\left(A_{2 n, j+1} x \cup A_{2 n+1, j+1} x\right), \\
A_{2 n, j+1} \oplus A_{2 n+1, j+1} & \text { otherwise. }
\end{array}\right.
$$

Where the algorithm starts at the bottom level of the tree by setting $A_{n, J}=B_{n, J}$ and searches its way upward from $j=J-1$ to 0 . The final basis $A_{0,0}$ will be the basis which minimizes the information cost function and is referred to as the best basis of the signal $f(t)$. Therefore, the best basis algorithm can effectively characterize the time frequency composition of a non-stationary signal. From the best basis algorithm a feature vector can be constructed from the energy function $E(\cdot)$ as it applies to those nodes which make up $A_{0,0}$. 
The construction of the feature vectors from the best basis approach is as follows. First, $f(t)$ is decomposed using the WPT. This results in a full decomposition tree. Next the best basis algorithm finds those nodes which minimize the cost function $E(\cdot)$. The feature vector $E$ is then defined as those entropy measures resulting from the nodes which make up the bases found from the best basis algorithm. The entropy measures of those nodes which are not a part of the best basis are taken to be 0 . This construction is given as,

$$
\mathbf{E}_{\text {bestbasis }}=\left[E_{1,1}, E_{1,2}, E_{2,1} \ldots E_{j, n}\right]
$$

such that,

$$
E_{j, n}= \begin{cases}\sum_{k} E\left(w_{j, k}^{n}\right) & \text { if } W_{j}^{n} \in A_{0,0} \\ 0 & \text { otherwise }\end{cases}
$$

Figure 3.5 shows an example of this feature vector construction for a third level decomposition where the filled nodes are those that make up the best basis found from the above algorithm.

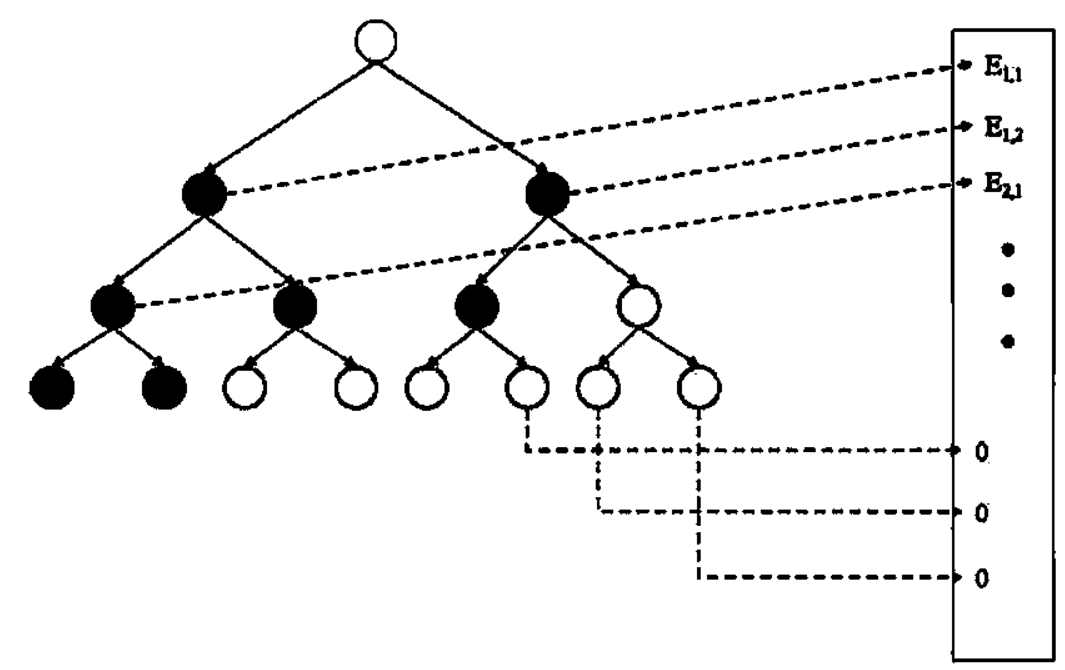

Figure 3.5: Wavelet packet feature representation based on nodes from best basis tree

Taking the entropy values of those nodes which do not contribute to the determined best basis does not imply this was their original entropy measure. Rather, this was done to keep 
the dimensionality of the feature vectors consistent while at the same time eliminating the contribution of those nodes not included in the best basis. 


\section{Chapter 4}

\section{Fault Detection and Identification Scheme}

This chapter describes in detail the proposed fault detection and identification scheme which uses support vector machines as the diagnosis machine and the wavelet packet transform for feature extraction. The first section describes a typical system for which this FDI scheme is applicable. The final section outlines the application of our FDI scheme.

\subsection{System for FDI Application}

To be able to build an FDI scheme, a system must first lend itself to a subsystem (component) decomposition. Consider a system $T$ which can be partitioned into its subsystem (component) representation. Figure 4.1 shows an idealized system which is partitioned into its various subsystems $S_{i}$ such that $T=\left\{S_{1}, S_{2} \ldots S_{n}\right\}$.

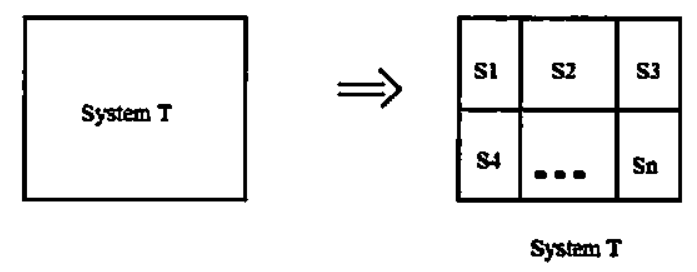

Figure 4.1: System T decomposed into its respective subsystems 
The goal of an FDI system in this setting is to first detect if a fault has occurred and then identify the most likely cause of the fault among the various subsystems $S_{i}$. Because, the approach developed is data driven the FDI scheme examines outputs of the system and use them to determine the location of the fault. Therefore, to obtain an understanding of the various fault conditions one needs to superficially introduce prescribed faults to each of the subsystems $S_{i}$, and observe the output behavior due to a set input. In other words, a "fault dictionary" is built by shifting subsystems beyond their designed limits, exciting the overall system and observing the response. Figure 4.2 outlines the process of pushing a subsystem beyond its limits and exciting it to produce an output response.

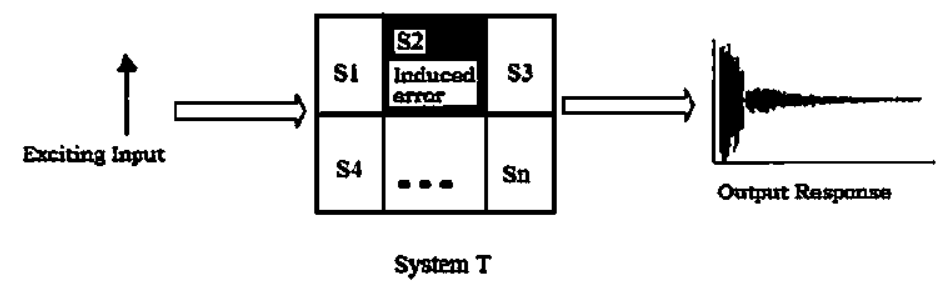

Figure 4.2: Example of a system's response to error

This process allows us to build the needed fault features in which to train and test a diagnosis machine. By introducing errors and exciting the system the hope is that the error will manifest itself in some form in the output response of the system. This narrows fault identification down to a process of extracting suitable features from the output response and using them to train a diagnosis machine. Figure 4.3 displays the process model for our proposed FDI system.

\subsection{Fault Diagnosis Model}

As mentioned before examination of the raw output of a system for fault detection and identification often leads to poor performance and inaccurate results. Rather, a fault's characteristic features will more likely be buried in the frequency content of the output response. Therefore, the wavelet packet transform as described in Chapter 3 is used to draw out those features tied up in the frequency spectrum of the signal. The features obtained at 


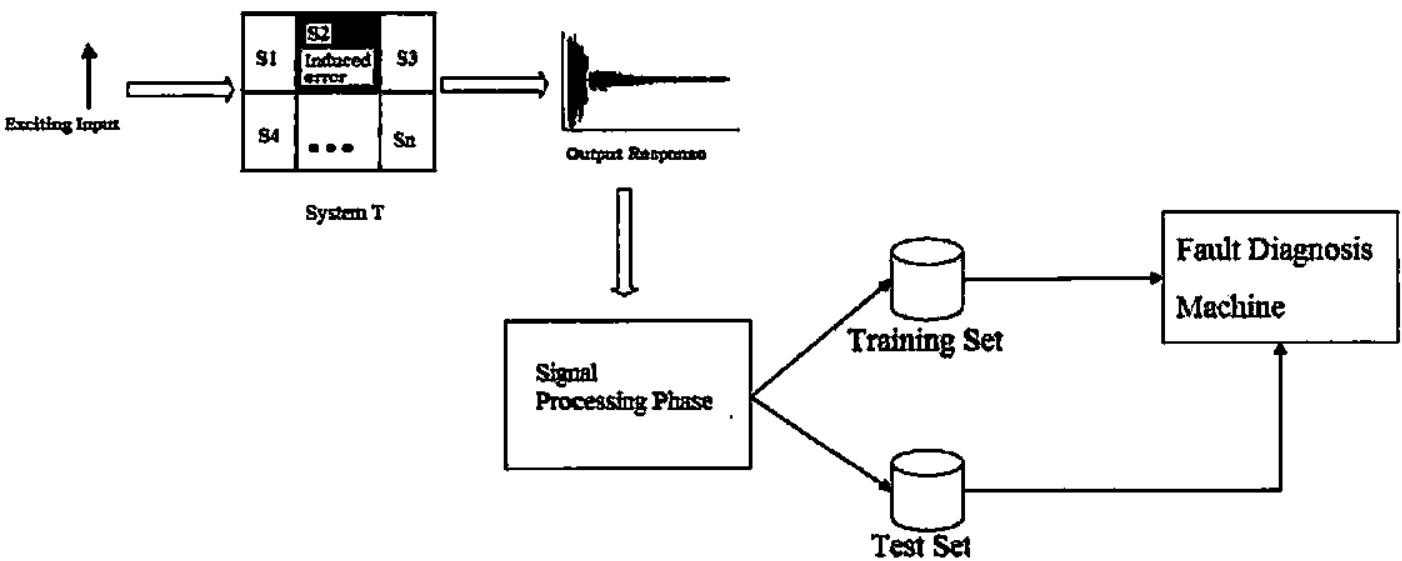

Figure 4.3: Block diagram of exciting a system in fault to collect training and testing data for FDI scheme.

the signal processing stage are in turn used to train the support vector machines for fault detection and identification purposes. The fault detection and isolation scheme is outlined as follows,

1. Signal Processing Phase: This phase takes those raw observations from the excited system and decomposes them using the wavelet packet transform into its full decomposition tree. The wavelet packets are used to calculate entropy measures for each of the nodes in the tree. The feature vector from the entropy metric is constructed in two ways:

(a) By using all nodes in the decomposition tree.

(b) Using only those nodes determined from the best basis algorithm

2. Fault Diagnosis Machine: This phase consists of support vector machines trained to detected and recognize patterns arising from each of the fault classes. The SVMs are trained on those features obtained from the signal processing phase. Training for multi-class pattern recognition problems is done in two ways as described in Chapter 2: 
(a) One-Versus-All with a winner takes all strategy

(b) One-Versus-One with a max votes wins strategy.

Figure 4.4 outlines the block diagram of the proposed FDI scheme.

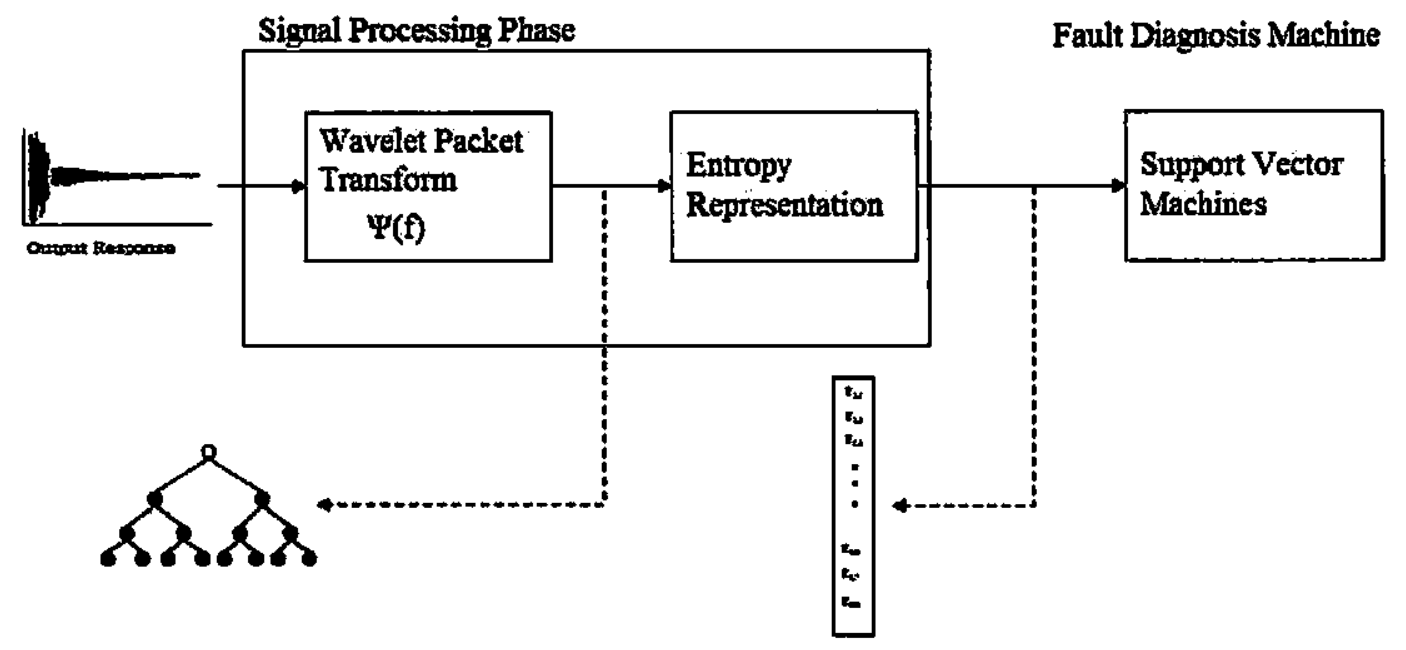

Figure 4.4: Expanded Block Diagram of Proposed FDI Scheme 


\section{Chapter 5}

\section{Simulation Results}

\subsection{System description}

In this chapter the proposed FDI scheme is applied to an Attitude-Control System of an Aircraft and a Sallen-Key Band-pass Filter.

\subsubsection{Attitude-Control System of an Aircraft}

In the first example the attitude-control system of an aircraft developed in [21] is considered. This system is used to control the position of the control flaps of a modem aircraft. Figure 5.1 gives the block diagram of one axis of the described system.

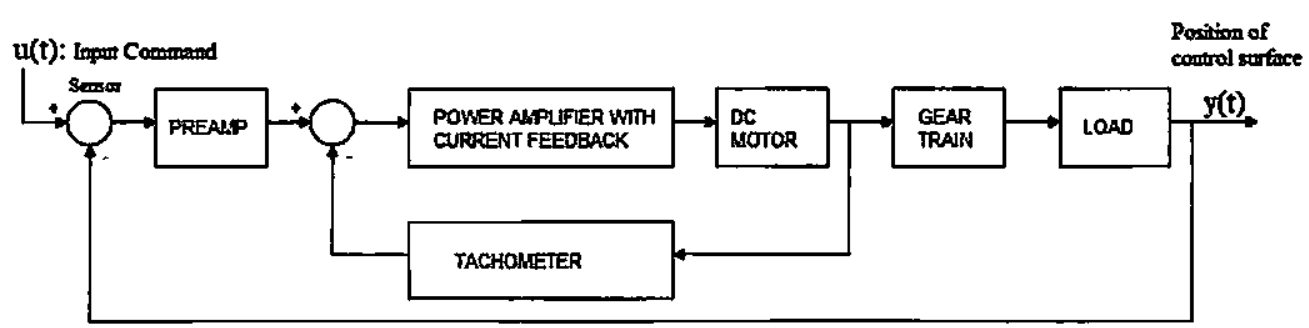

Figure 5.1: Block diagram of the attitude-control system

As with most modern aircraft the flight controls are driven by electronic actuators with their corresponding electronic controls. It is identified that the driving and the more critical component of this system is the DC motor. Therefore, the FDI system proposed will detect 
and identify errors within the DC motor portion of the system. More specifically, the FDI system is designed to recognize faults in the armature inductance and resistance of the DC motor by examination of the entire system's output $y(t)$. Figure 5.2 displays the transfer function block diagram of the examined system. From Figure 5.3 the DC motor's contribution to the overall system is seen.

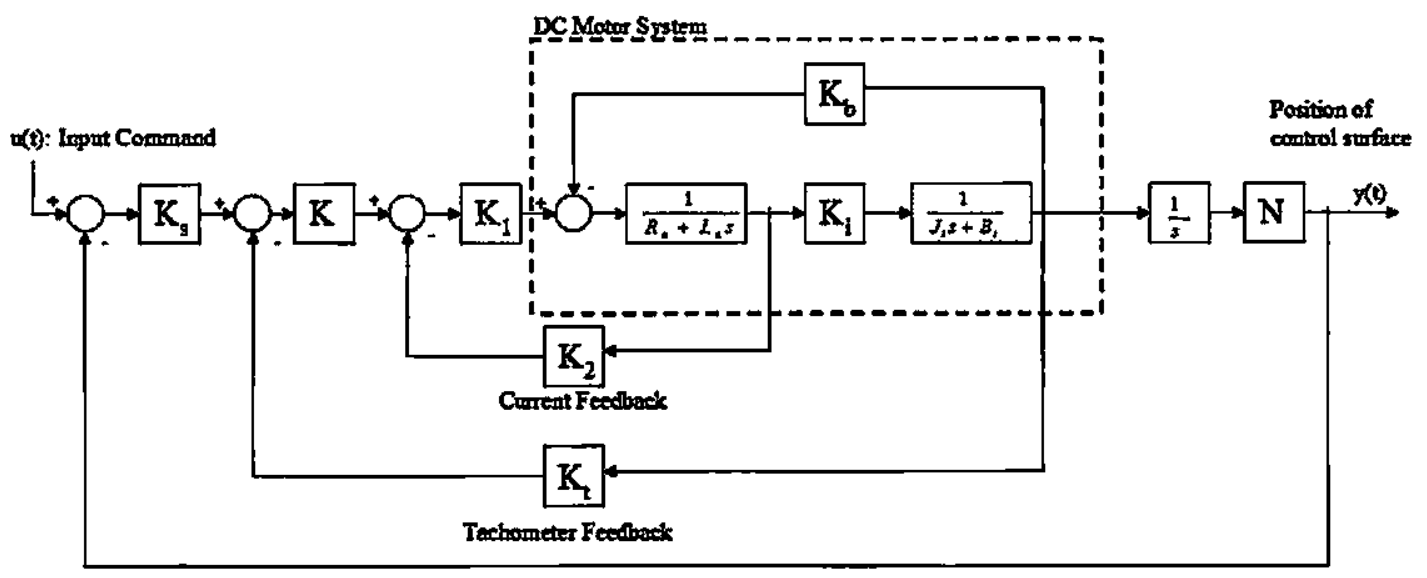

Figure 5.2: Transfer Function Block diagram of the attitude-control system

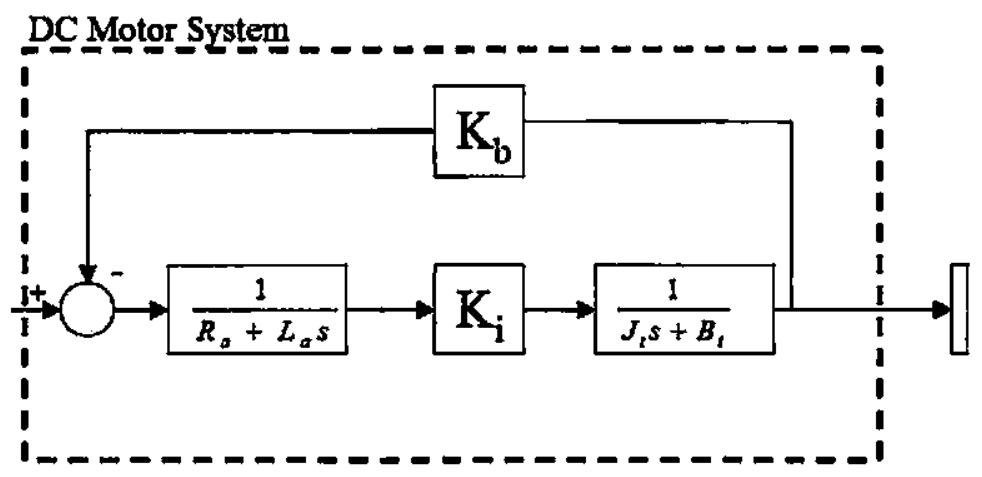

Figure 5.3: Expanded view of DC Motor's Transfer Function Block Diagram

The dynamics of the a attitude-control system can be obtained by examination of the the transfer function block diagram in Fig. 5.2.The open-loop transfer function is found 
using Mason's gain formula:

$$
\begin{gathered}
G(s)=\frac{Y(s)}{\Theta_{e}(s)} \\
=\frac{K_{s} K_{1} K_{i} K N}{s\left[L_{a} J_{t} s^{2}+\left(R_{a} J_{t}+L_{a} B_{t}+K_{1} K_{2} J_{t}\right) s+R_{a} B_{t}+K_{1} K_{2} B_{t}+K_{i} K_{b}+K K_{1} K_{t} K_{i}\right]}
\end{gathered}
$$

The closed loop response is found using:

$$
M(s)=\frac{G(s)}{1+G(s) H(s)}
$$

Where the variables and their respective values are given in Table 5.1.

Table 5.1: Attitude-Control System Component Values

\begin{tabular}{c|c|c}
\hline Symbol & Description & Value \\
\hline$K_{s}$ & Gain of encoder & $1 \mathrm{~V} / \mathrm{rad}$ \\
$K$ & Gain of preamplifier & $181.17 \mathrm{~V} / \mathrm{V}$ \\
$K_{1}$ & Gain of power amplifier & $10 \mathrm{~V} / \mathrm{V}$ \\
$K_{2}$ & Gain of current feedback & $0.5 \mathrm{~V} / \mathrm{A}$ \\
$K_{t}$ & Gain of tachometer feedback & $0 \mathrm{~V} / \mathrm{rads} / \mathrm{s}$ \\
$K_{i}$ & Torque constant of motor & $9.0 \mathrm{oz}-\mathrm{in} . / \mathrm{A}$ \\
$K_{b}$ & Back-emf constant of motor & $0.0636 \mathrm{~V} / \mathrm{rads} / \mathrm{s}$ \\
$J_{m}$ & Inertia of motor rotor & $0.0001 \mathrm{oz}-\mathrm{in} .-\mathrm{s}^{2}$ \\
$J_{L}$ & Inertia of load & $0.01 \mathrm{oz}-\mathrm{in} . \mathrm{s}^{2}$ \\
$B_{m}$ & Viscous-friction coefficient of motor & $0.005 \mathrm{oz}-\mathrm{in} .-\mathrm{s}$ \\
$B_{L}$ & Viscous-friction coefficient of load & $1.0 \mathrm{oz}-\mathrm{in} .-\mathrm{s}$ \\
$N$ & Gear-train ratio & $1 / 10$ \\
$R_{a}$ & Armature Resistance & $5 \Omega$ \\
$L_{a}$ & Armature Inductance & $0.003 \mathrm{H}$ \\
\hline
\end{tabular}

\subsubsection{Sallen-Key Band-pass Filter}

The second system considered is a Sallen-Key band-pass filter with a center frequency of $25 \mathrm{kHz}$ as shown in Figure 5.4. This system has also been used for evaluation purposes in other fault diagnosis schemes presented in [1, 25]. The proposed FDI scheme focuses on faults arising in the resistors and capacitors that comprise this circuit. Specifically, the FDI scheme will be able to detect and identify faults in resistors $R_{1}, R_{2}, R_{3}$ and the two capacitors $C_{1}, C_{2}$. 


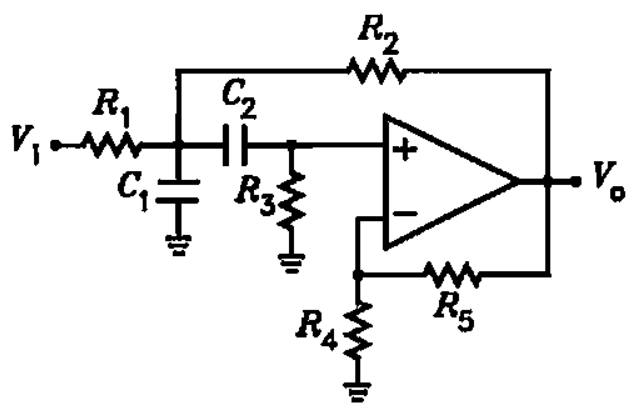

Figure 5.4: Sallen-Key bandpass filter with center frequency of $25 \mathrm{kHz}$

The transfer function for the examined filter is obtained as follows. The voltage-gain transfer function is given by

$$
\frac{V_{o}}{V_{i}}=K \frac{(1 / Q)\left(s / \omega_{0}\right)}{\left(s / \omega_{o}\right)^{2}+(1 / Q)\left(s / \omega_{0}\right)+1}
$$

The gain $K$ at the resonant frequency $\omega_{0}$ is given as,

$$
K=\frac{R_{2}}{R_{1}+R_{2}} \times \frac{K_{o} R_{3} C_{2}}{\left(R_{1} \| R_{2}\right)\left(C_{1}+C_{2}\right)+R_{3} C_{2}\left[1-K_{o} R_{1} /\left(R_{1}+R_{2}\right)\right]}
$$

where the resonant frequency $\omega_{0}$ is found as,

$$
\omega_{0}=\frac{1}{\sqrt{\left(R_{1}|| R_{2}\right) R_{3} C_{1} C_{2}}}
$$

The quality factor is found to be,

$$
Q=\frac{\sqrt{\left(R_{1} \| R_{2}\right) R_{3} C_{1} C_{2}}}{\left(R_{1} \| R_{2}\right)\left(C_{1}+C_{2}\right)+R_{3} C_{2}\left[1-K_{o} R_{1} /\left(R_{1}+R_{2}\right)\right]}
$$

and the gain from non-inverting op-amp is given as,

$$
K_{o}=1+\frac{R_{5}}{R_{4}}
$$

The resistor and capacitor values are are given in Table 5.2. 
Table 5.2: Resistor and Capacitor Values for the Sallen-Key Bandpass Filter with center frequency of $25 \mathrm{kHz}$

\begin{tabular}{c|c}
\hline Component & Nominal value \\
\hline$R_{1}$ & $5.18 \mathrm{k} \Omega$ \\
$R_{2}$ & $1 \mathrm{k} \Omega$ \\
$R_{3}$ & $2 \mathrm{k} \Omega$ \\
$R_{4}$ & $4 \mathrm{k} \Omega$ \\
$R_{5}$ & $4 \mathrm{k} \Omega$ \\
$C_{1}$ & $5 \mathrm{nF}$ \\
$C_{2}$ & $5 \mathrm{nF}$ \\
\hline
\end{tabular}

\subsection{FDI performance evaluation}

\subsubsection{Attitude-Control System of an Aircraft case}

To evaluate the performance for the proposed FDI scheme as it applies to the DC motor of the attitude-control System, the armature resistance $R_{a}$ and inductance $L_{a}$ are assumed to have a tolerance of $\pm 5 \%$. The other components which make up the DC motor are assumed to have a tolerance of $\pm 1 \%$. This investigation considers the single fault model for errors. Where in the single fault model, failures are assumed to originate from a single component and not as a combination of component failures. A fault in $R_{a}$ or $L_{a}$ is considered to be any deviation outside their prescribed tolerance limits. The nominal values and respective tolerances of the components for the DC motor found in the attitude-control system are given in Table 5.3.

Table 5.3: Component and Tolerance Values for DC Motor of the Attitude-Control System

\begin{tabular}{|c|c|c|}
\hline Component & Nominal value & Tolerance \\
\hline$R_{a}$ & $5 \Omega$ & $\pm 5 \%$ \\
\hline$L_{a}$ & $0.003 \mathrm{H}$ & $\pm 5 \%$ \\
\hline$K_{i}$ & $9.0 \mathrm{oz}-\mathrm{in} . / \mathrm{A}$ & $\pm 1 \%$ \\
\hline$K_{b}$ & $0.0636 \mathrm{~V} / \mathrm{rads} / \mathrm{s}$ & $\pm 1 \%$ \\
\hline$J_{m}$ & $0.0001 \mathrm{oz}-$ in.-s $^{2}$ & $\pm 1 \%$ \\
\hline$B_{m}$ & $0.005 \mathrm{oz}-\mathrm{in} . \mathrm{s}$ & $\pm 1 \%$ \\
\hline
\end{tabular}

To build the needed training and testing samples for each of the fault and nominal classes a Monte Carlo approach is followed. To construct samples for the two fault classes the 
respective faulty component values are randomly drawn from the uniform distribution defined on the intervals $\left[0.1 X_{n} ;(1-t) X_{n}\right]$ and $\left[(1+t) X_{n} ; 1.9 X_{n}\right]$ where $t$ is the tolerance range for the $X_{n}$ component. The other component values are drawn randomly from their defined tolerance intervals. The system is then excited using a step input for $u(t)$ and the output response is noted from $y(t)$. The nominal class is constructed by allowing each of the component values to vary randomly within their tolerance limits and the system is again excited using a step input for $u(t)$ and the output response observed from $y(t)$. The summary of the two fault classes and one nominal class is given below,

1. Faulty $R_{a}$ : Faulty $R_{a}$ samples drawn from interval $[0.5 \Omega, 4.75 \Omega] \cup[5.25 \Omega, 9.5 \Omega]$ other components drawn from their tolerance interval defined by the tolerance limits.

2. Faulty $L_{a}$ : Faulty $L_{a}$ samples drawn from interval $\left[3 * 10^{-4} \mathrm{H}, 2.85 * 10^{-3} \mathrm{H}\right] \cup[3.15 *$ $\left.10^{-3} \mathrm{H}, 5.7 * 10^{-3} \mathrm{H}\right]$ other components drawn from their tolerance interval defined by the tolerance limits.

3. Nominal Operation Mode: All components drawn from within their tolerance limits.

Outputs observed from the various conditions are preprocessed using the wavelet packet methods and feature representation described in Chapter 3. In this investigation the wavelet packet transform uses a fourth order Daubechies mother wavelet with a decomposition depth of 8 . A decomposition to a depth of 8 yields feature vectors with a dimension of 510 . The two described multi-class extension methods for SVMs along with the Gaussian kernel function are used to build the fault diagnosis machine. In each experiment 100 training samples and 200 testing samples are generated for each class (fault,nominal) Giving a total of 300 training samples and 600 testing samples for a given training and testing iteration. For numerical stability this procedure is repeated for 32 iterations and the generalization errors are averaged. To obtain a good regularization parameter $C$ and appropriate kernel value $\gamma$ for Gaussian kernel function their effect on the generalization performance (test error) is examined. A detailed analysis of the proposed FDI scheme is based on the regularization and kernel parameter combination which yields the best performance.

The results are reported as follows: 
1. Performance of FDI scheme when feature vectors consist of all node entropy measures (Eqns. (3.4.4),(3.4.5))

(a) Applied to One-Verses-One SVM multi-class extension

(b) Applied to One-Verses-All SVM multi-class extension

2. Performance of FDI scheme where feature vectors consist of the best basis node entropy measures (Eqns. (3.4.8),(3.4.9))

(a) Applied to One-Verses-One SVM multi-class extension

(b) Applied to One-Verses-All SVM multi-class extension

3. Detailed evaluation of FDI scheme based on the best regularization and gaussian kemel combination.

\section{Features: All node entropy measures}

This section presents the results for the FDI scheme when the feature vectors are constructed from the entropy measure applied to all nodes in the wavelet packet tree. Each of the results are broken down by training type (One-verses-One, One-verses-All). Figure 5.5 displays the performance of the One-verses-One multi-class extension for SVMs. Each of the curves presented in Fig. 5.5 represent a fixed value for the Gaussian kernel parameter with a varying regularization parameter. Figure 5.5 shows that good generalization performance is achieved when the Gaussian kernel parameter is 0.1 and the regularization value is 1900 . The minimum misclassification error of this combination of parameter values is $5.1 \%$.

Figure 5.6 displays the performance of the One-verses-All multi-class extension for SVMs. The curves shown in Figure 5.6 reflect the generalization performance for the various fixed Gaussian kernel parameters as the regularization parameter is varied. As in the previous performance evaluation the best generalization performance is achieved when the Gaussian kernel parameter is 0.1 for an associated regularization value of 300 . The generalization error achieved with this combination of parameter values is found to be $4.4 \%$. 


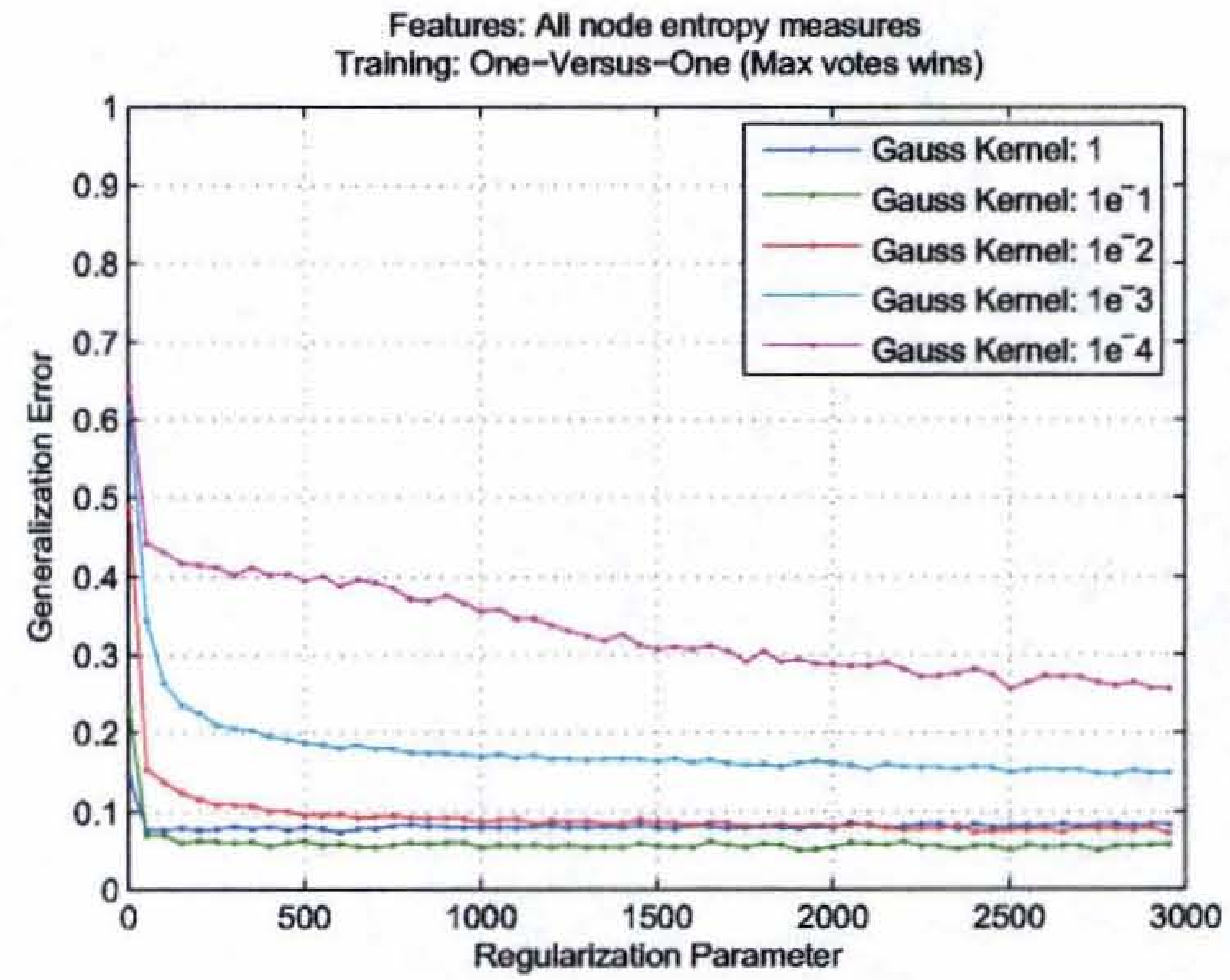

Figure 5.5: Attitude-Control System FDI Generalization Error for One-verse-One Training with All Nodes for Features

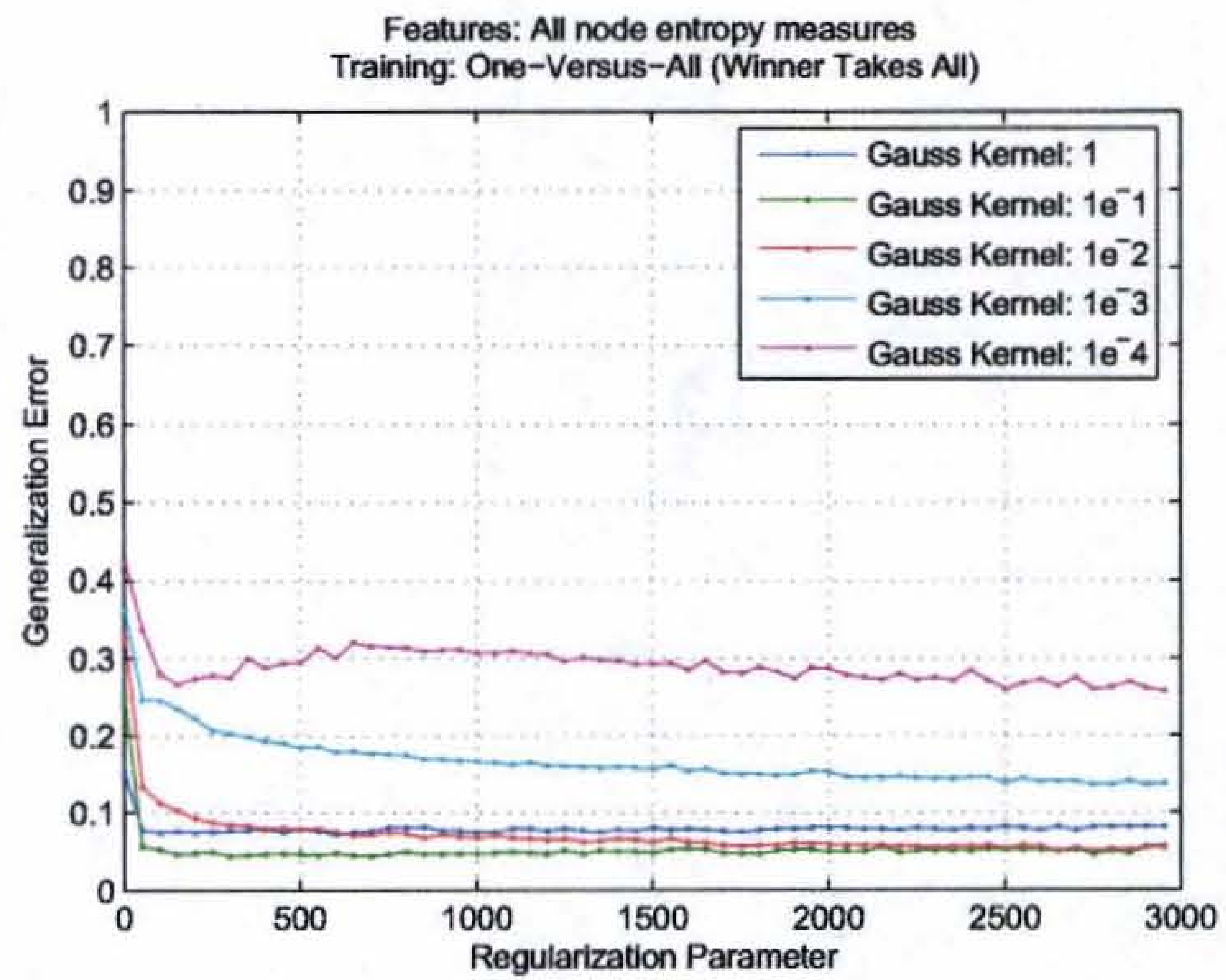

Figure 5.6: Attitude-Control System FDI Generalization Error for One-verse-All Training with All Nodes for Features 
Since the best performance is achieved by the same fixed Gaussian kernel parameter a comparison of the two associated generalization curves is done to see if there is any clear difference in the two multi-class training methods. The comparison of the two curves are displayed in Figure 5.7. Examination of Figure 5.7 shows that the One-verse-All extension performs only slightly better than the One-verses-One. Table 5.4 gives a detailed performance analysis for each of the training extensions. Again, the details in Table 5.4 are based on the best combination of Gaussian and regularization kemel parameters.

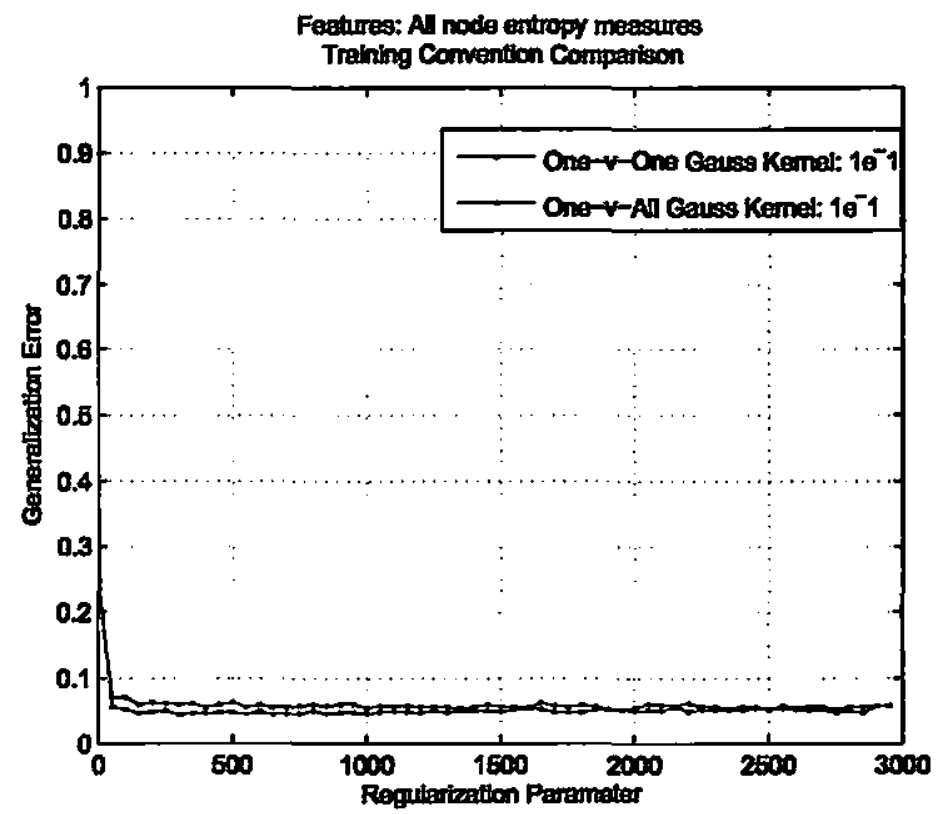

Figure 5.7: Attitude-Control System FDI Generalization Error. Best Curve Comparison of two Training Conventions with All Nodes as Features

Features: Best basis node entropy measures

This section presents the results for the FDI scheme when the feature vectors are constructed from the entropy measure applied to those nodes left from the best basis algorithm. Each of the results are broken down by training type (One-verses-One, One-versesAll). Figure 5.8 displays the performance of the One-verses-One multi-class extension for 
Table 5.4: Best Performance Breakdown: All Node Packets

\begin{tabular}{|c|c|c|}
\hline Fault Classes & $\begin{array}{c}\text { One-Versus-One } \\
\text { Error (\%) } \\
(C, \gamma)=(1900,0.1)\end{array}$ & $\begin{array}{c}\text { One-Versus-All } \\
\text { Error (\%) } \\
(C, \gamma)=(300,0.1)\end{array}$ \\
\hline No Fault & 0.14 & 0.03 \\
\hline Faulty $R_{a}$ & 2.94 & 1.82 \\
\hline Faulty $L_{a}$ & 2.01 & 2.57 \\
\hline Total Error $(\%)$ & 5.09 & 4.43 \\
\hline
\end{tabular}

SVMs. The curves presented in Figure 5.8 represent a series of fixed values for the Gaussian kernel parameter with a varying regularization parameter. Examination of Figure 5.8 shows good generalization performance is achieved when the Gaussian kernel parameter is 0.1 and the regularization parameter value is 1350 . This combination of parameter values yields a minimum generalization error of $2.7 \%$.

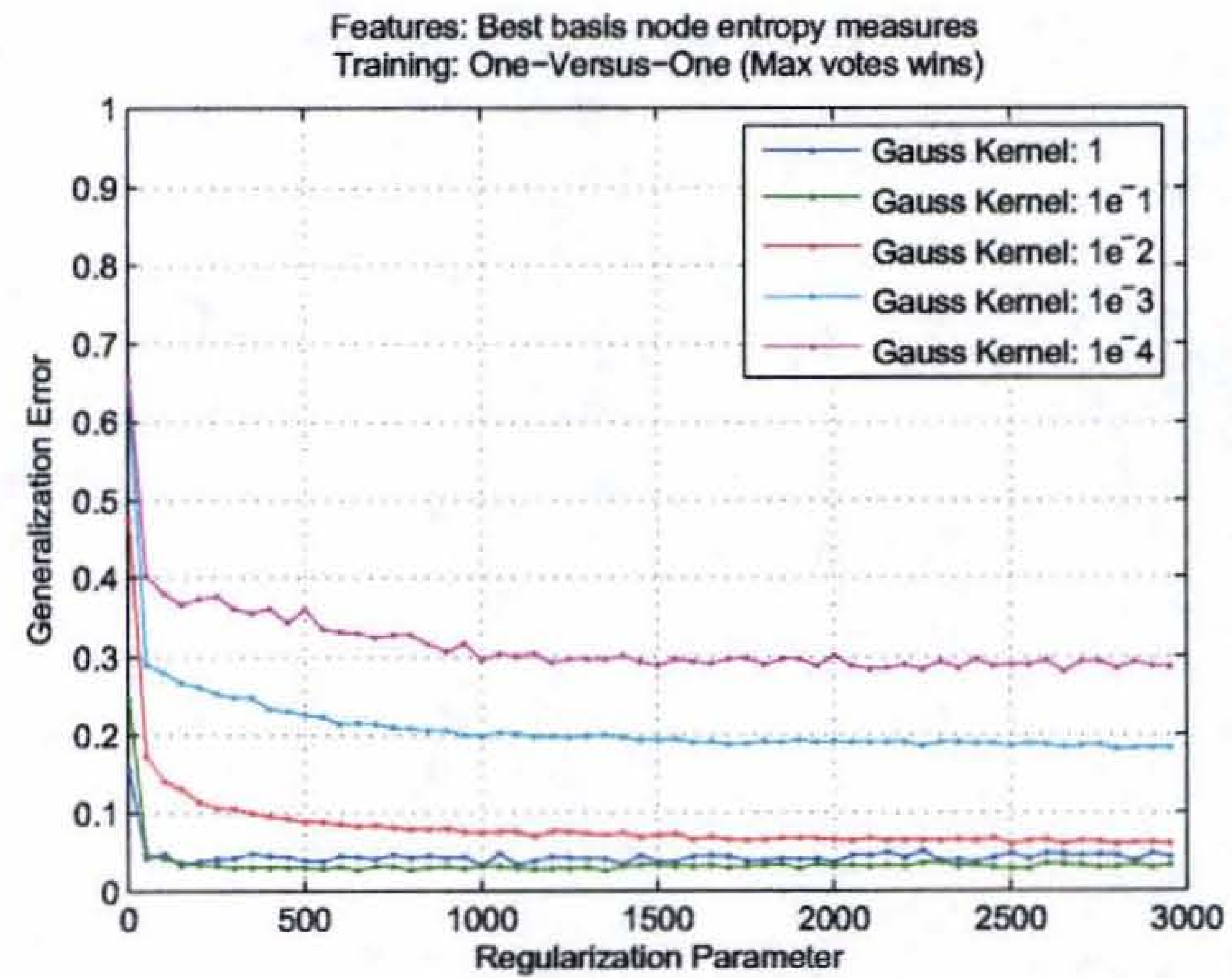

Figure 5.8: Attitude-Control System FDI Generalization Error for One-verse-One Training with Best Basis Nodes as Features

Figure 5.9 displays the performance of the One-verses-All multi-class extension for SVMs. The curves shown in Fig. 5.9 reflect the generalization performance for the various fixed 
Gaussian kernel parameters as the regularization parameter is varied. The best generalization performance is achieved when the Gaussian kernel parameter is 0.1 with associated regularization parameter value of 1700 . The generalization error achieved with this combination of parameter values is $2.5 \%$

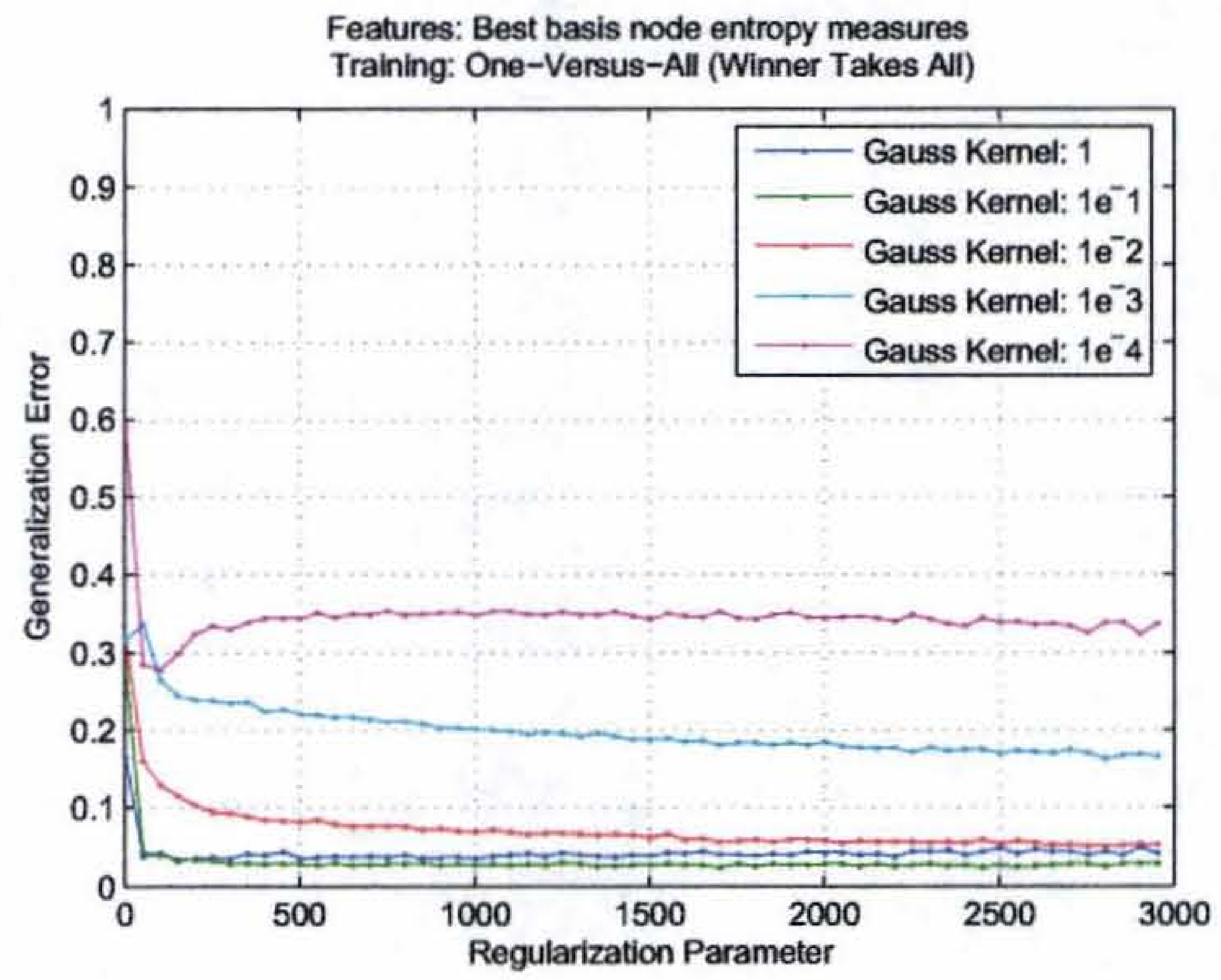

Figure 5.9: Attitude-Control System FDI Generalization Error for One-verse-All Training with Best Basis Nodes as Features

Since the best performance is achieved by the same fixed Gaussian kernel parameter comparison of the two associated generalization curves is done to determined if there is any clear difference in performance. The comparison of the two curves is displayed in Figure 5.10 and shows no clear performance differences in the two training methods. A detailed comparison of the best performance from each training convention is given in Table 5.5.

Table 5.6 summarizes the best performances from each of the feature representation methods. It can be seen that the fault diagnosis machine based on features from the best basis algorithm out performs one that is trained on all node entropy measures. This is most likely due to the fact that the best basis algorithm keeps only those nodes which significantly contribute to the representation of a signal. While, there was a difference seen 


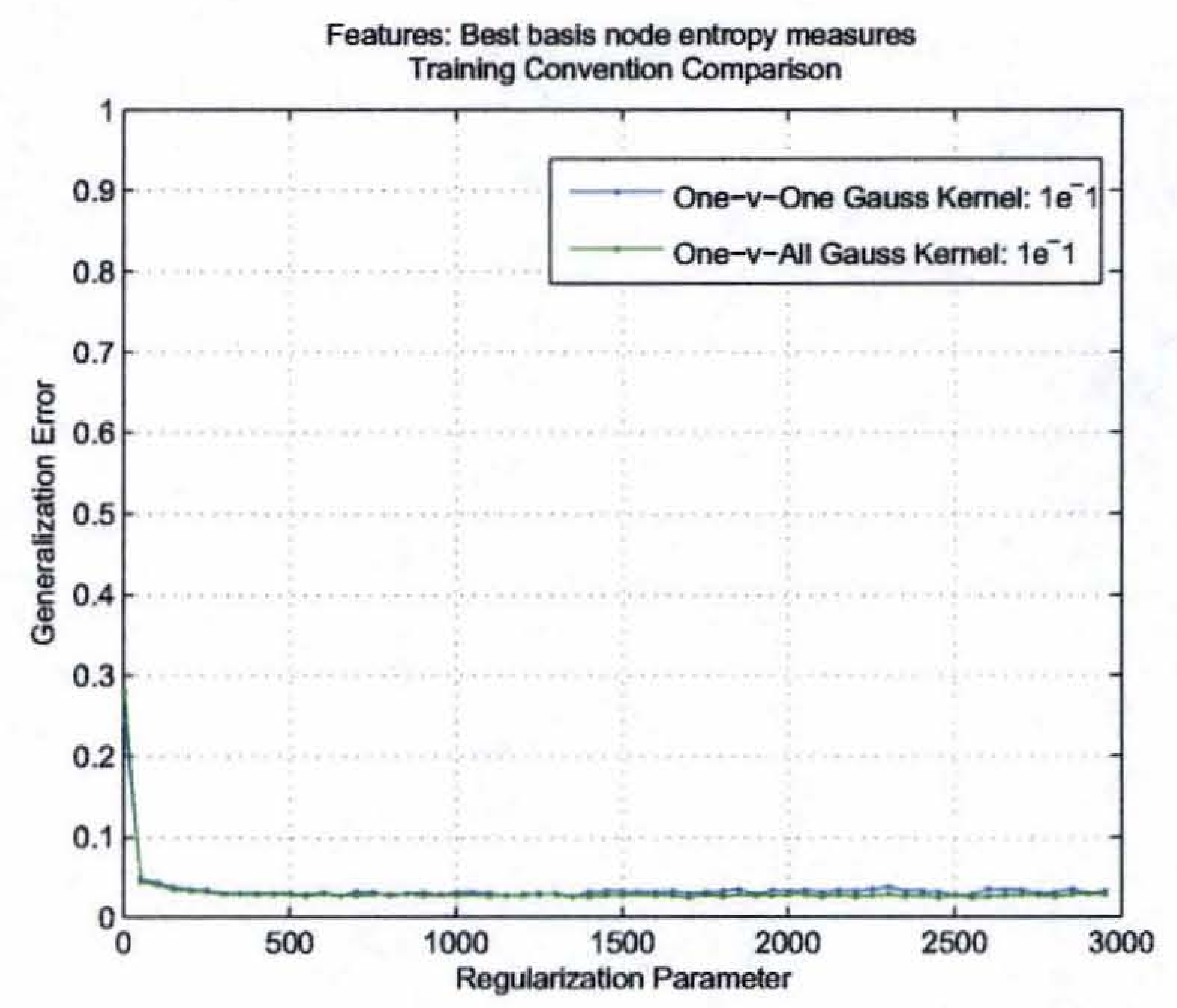

Figure 5.10: Attitude-Control System FDI Generalization Error. Best Curve Comparison of two Training Conventions with Best Basis Nodes as Features

Table 5.5: Best Performance Breakdown: Best Tree Node Packets

\begin{tabular}{|c|c|c|}
\hline Fault Classes & $\begin{array}{c}\text { One-Versus-One } \\
\text { Error (\%) } \\
(C, \gamma)=(1350,0.1)\end{array}$ & $\begin{array}{c}\text { One-Versus-All } \\
\text { Error (\%) } \\
(C, \gamma)=(1700,0.1)\end{array}$ \\
\hline No Fault & 0.09 & 0.08 \\
\hline Faulty $R_{a}$ & 1.73 & 1.49 \\
\hline Faulty $L_{a}$ & 0.83 & 0.87 \\
\hline Total Error (\%) & 2.67 & 2.45 \\
\hline
\end{tabular}


in performance with regard to the feature extraction methods there is no real difference in the performance of the two training techniques.

Table 5.6: Comparison of the Two Feature Representation Methods Best Performance

\begin{tabular}{c|cc|cc} 
& \multicolumn{2}{|c|}{ One-Versus-One } & \multicolumn{2}{c}{ One-Versus-All } \\
& $(C, \gamma)$ & Error $(\%)$ & $(C, \gamma)$ & Error (\%) \\
\hline $\begin{array}{c}\text { All Node Packets used } \\
\text { for Feature Representation }\end{array}$ & $(1900,0.1)$ & 5.09 & $(300,0.1)$ & 4.43 \\
\hline $\begin{array}{c}\text { Best Basis Node Packets used } \\
\text { for Feature Representation }\end{array}$ & $(1350,0.1)$ & 2.67 & $(1700,0.1)$ & 2.45 \\
\hline
\end{tabular}

\subsubsection{Sallen-Key Band-pass Filter case}

To test the FDI scheme on a more complex system the Sallen-Key Bandpass filter developed in section 5.1.2 is examined. This system has been studied in other fault diagnosis methods which usually involve a feed forward neural network as the diagnosis machine $[25,1]$. The emphasis in the past efforts focused on preprocessing methods to control the dimensionality of the features vectors. This is in turn regulated the capacity of their neural networks. As discussed in Chapter 2 the dimension of the feature vectors does not affect the capacity of support vector machines. In contrast, SVMs readily accepts high dimensional features. The capacity of SVMs is controlled by the number of support vectors used to determine the margin. In the evaluation of the proposed FDI scheme for the Sallen-Key Band-pass Filter the fault classes are comprised of the resistors $R_{1}, R_{2}, R_{3}$ and capacitors $C_{1}, C_{2}$. In particular this FDI scheme is designed to detect and identify errors arising in the above considered components. Faults occurring in $R_{4}$ and $R_{5}$ form an ambiguity class and can be lumped into a single fault class denoted as "gain fault." This fault class can be detected by a simple dc measurement, and it is not included in this analysis [1]. A fault in a component is defined as any deviation outside its nominal value by $+1-50 \%$. This definition of a fault follows the work investigated by [1] and [25]. The nominal values and respective tolerances of the components for the Sallen-Key bandpass filter with a center frequency of $25 \mathrm{kHz}$ are given in Table 5.7. 
Table 5.7: Component Values and Tolerance Ranges for the Sallen-Key Bandpass Filter

\begin{tabular}{|c|c|c|}
\hline Component & Nominal value & Tolerance \\
\hline$R_{1}$ & $5.18 \mathrm{k} \Omega$ & $\pm 5 \%$ \\
\hline$R_{2}$ & $1 \mathrm{k} \Omega$ & $\pm 5 \%$ \\
\hline$R_{3}$ & $2 \mathrm{k} \Omega$ & $\pm 5 \%$ \\
\hline$R_{4}$ & $4 \mathrm{k} \Omega$ & $\pm 5 \%$ \\
\hline$R_{5}$ & $4 \mathrm{k} \Omega$ & $\pm 5 \%$ \\
\hline$C_{1}$ & $5 \mathrm{nF}$ & $\pm 10 \%$ \\
\hline$C_{2}$ & $5 \mathrm{nF}$ & $\pm 10 \%$ \\
\hline
\end{tabular}

To build the needed training and testing samples for each of the fault and nominal classes a Monte Carlo approach is followed. This investigation considers the single fault model for errors. Where in the single fault model, failures are assumed to originate from a single component and not as a combination of component failures. To construct samples for the five fault classes the respective faulty component values are randomly drawn from the uniform distribution defined on the intervals $\left[0.1 X_{n} ; 0.5 X_{n}\right]$ and $\left[1.5 X_{n} ; 1.9 X_{n}\right]$ where $X_{n}$ is nominal component value the other component values are drawn randomly from their defined tolerance intervals. The system is excited using a impulse input for $V_{i}$ and the output response is noted from $V_{o}$. The nominal class is constructed by allowing each of the component values to vary randomly within their tolerance limits and the system is again excited using a impulse input at $V_{i}$ and the output response is observed from $V_{o}$. The summary of the five fault classes and one nominal class is given below,

1. Faulty $R_{1}$ : Faulty $R_{1}$ samples are drawn from the interval:

$[0.518 k \Omega, 2.59 k \Omega] \cup[7.77 k \Omega, 9.842 k \Omega]$

All other components are drawn from within their tolerance interval defined by the tolerance limits.

2. Faulty $R_{2}$ : Faulty $R_{2}$ samples are drawn from the interval:

$[0.10 k \Omega, 0.50 k \Omega] \cup[1.50 k \Omega, 1.90 k \Omega]$

All other components are drawn from within their tolerance interval defined by the tolerance limits. 
3. Faulty $R_{3}$ : Faulty $R_{3}$ samples are drawn from the interval:

$[0.20 \mathrm{k} \Omega, 1.00 \mathrm{k} \Omega] \cup[3.00 \mathrm{k} \Omega, 3.80 \mathrm{k} \Omega]$

All other components are drawn from within their tolerance interval defined by the tolerance limits.

4. Faulty $C_{1}$ : Faulty $C_{1}$ samples are drawn from the interval:

$[0.5 n F, 2.5 n F] \cup[7.5 n F, 9.5 n F]$

All other components are drawn from within their tolerance interval defined by the tolerance limits.

5. Faulty $C_{2}$ : Faulty $C_{2}$ samples are drawn from the interval:

$[0.5 n F, 2.5 n F] \cup[7.5 n F, 9.5 n F]$

All other components are drawn from within their tolerance interval defined by the tolerance limits.

6. Nominal Operation Mode: All components drawn from within their tolerance limits.

Outputs observed from the various conditions are preprocessed using the wavelet packet methods and feature representation described in Chapter 3. In this investigation the wavelet packet transform uses a fourth order Daubechies mother wavelet with a decomposition depth of 8. A decomposition to a depth of 8 yields feature vectors with a dimensionality of 510. The two described multi-class extension methods for SVMs along with the Gaussian kernel function are used to build the fault diagnosis machine. In each experiment 100 training samples and 200 testing samples are generated for each class (fault,nominal) Giving a total of 600 training samples and 1200 testing samples for a given training and testing iteration. For numerical stability this procedure is repeated for 32 iterations and the generalization errors are averaged. In the first section of analysis additive white noise at an SNR of $30 \mathrm{~dB}$ is superimposed to the circuit's output before preprocessing. The results from this analysis are used to determine the best combination of regularization and gaussian kernel parameters based on generalization error performance (test error). Once the appropriate regularization and kernel parameters are found the output $V_{o}$ is subjected to various noise levels and the FDI performance is evaluated under noisy conditions.

The results are reported as follows: 
1. Performance of FDI scheme when feature vectors consists of all node entropy measures (Eqns. (3.4.4),(3.4.5)). With an SNR of $30 \mathrm{~dB}$ for $V_{0}$

(a) Applied to One-Verses-One SVM multi-class extension

(b) Applied to One-Verses-All SVM multi-class extension

2. Performance of FDI scheme where feature vectors consists of best basis node entropy measures (Eqns. (3.4.8),(3.4.9)). With an SNR of $30 \mathrm{~dB}$ for $V_{o}$

(a) Applied to One-Verses-One SVM multi-class extension

(b) Applied to One-Verses-All SVM multi-class extension

3. Detailed evaluation of FDI scheme based on best regularization and gaussian kernel combination. With an SNR of $30 \mathrm{~dB}$ for $V_{0}$

4. Evaluation of FDI scheme when output $V_{o}$ is subjected to varying noise conditions.

\section{Features: All node entropy measures}

This section presents the results when the FDI scheme is trained on feature vectors which are composed of the entropy measures from all the wavelet packet nodes. Each of the results are broken down by training type (One-verses-One, One-verses-All). Figure 5.11 displays the performance of the One-verses-One multi-class extension for SVMs. Where each of the curves presented in Fig. 5.11 represent a fixed value for the Gaussian kernel parameter for a varying regularization parameter. From Figure 5.11 good generalization performance for is achieved when the Gaussian kernel parameter is $1 * 10^{-5}$ and the regularization parameter value is 1200 . The generalization performance for this combination of parameters is $10.9 \%$.

Figure 5.12 displays the performance of the One-verses-All multi-class extension for SVMs. The curves shown in Fig. 5.12 reflect the generalization performance for the various fixed Gaussian kernel parameters as the regularization parameter is varied. The best generalization performance is achieved when the Gaussian kernel parameter is $1 * 10^{-5}$ with associated regularization of 2300 . This combinations yields a generalization error of $11.4 \%$. 


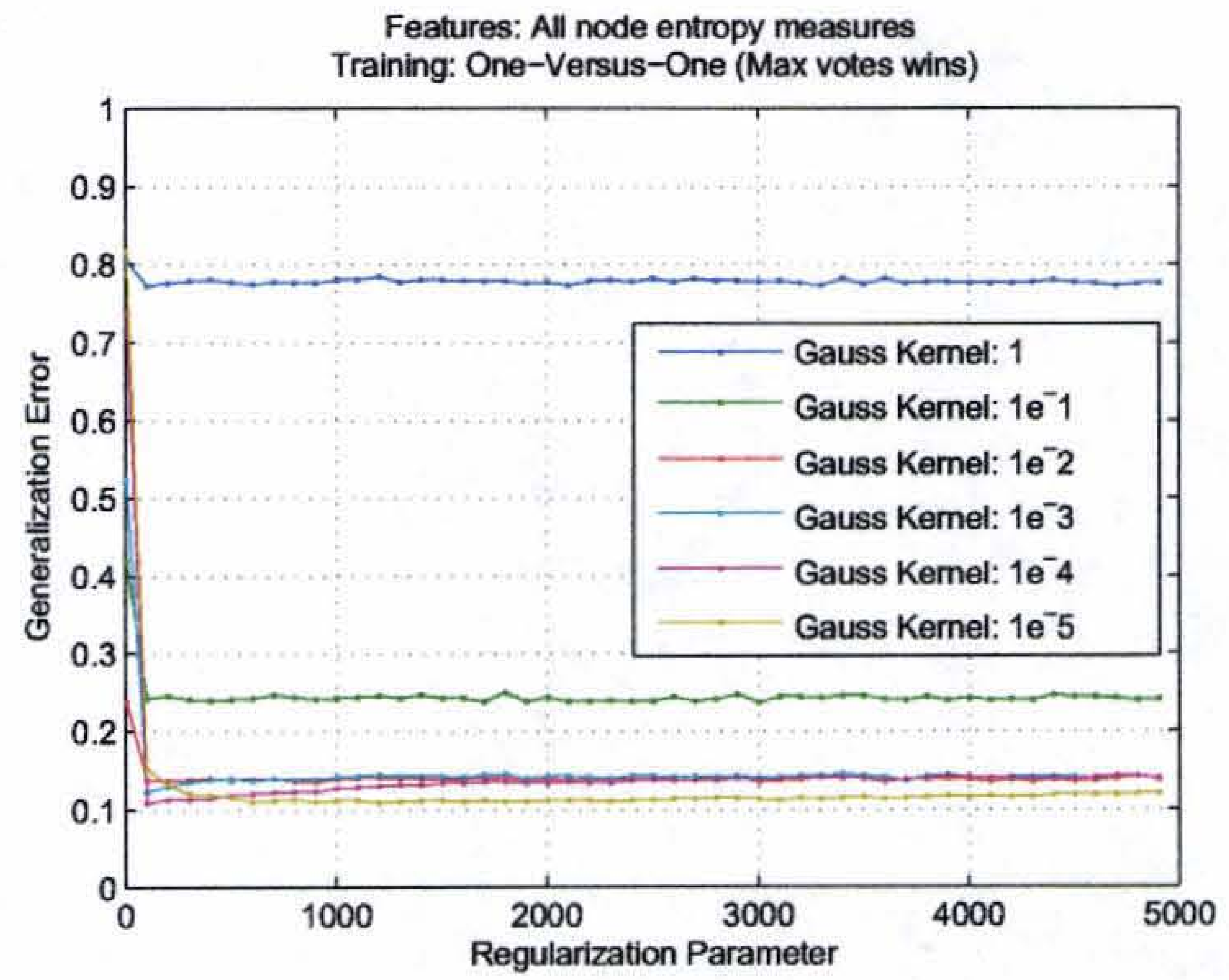

Figure 5.11: Sallen Key Bandpass FDI Generalization Error for One-verse-One Training with All Nodes for Features

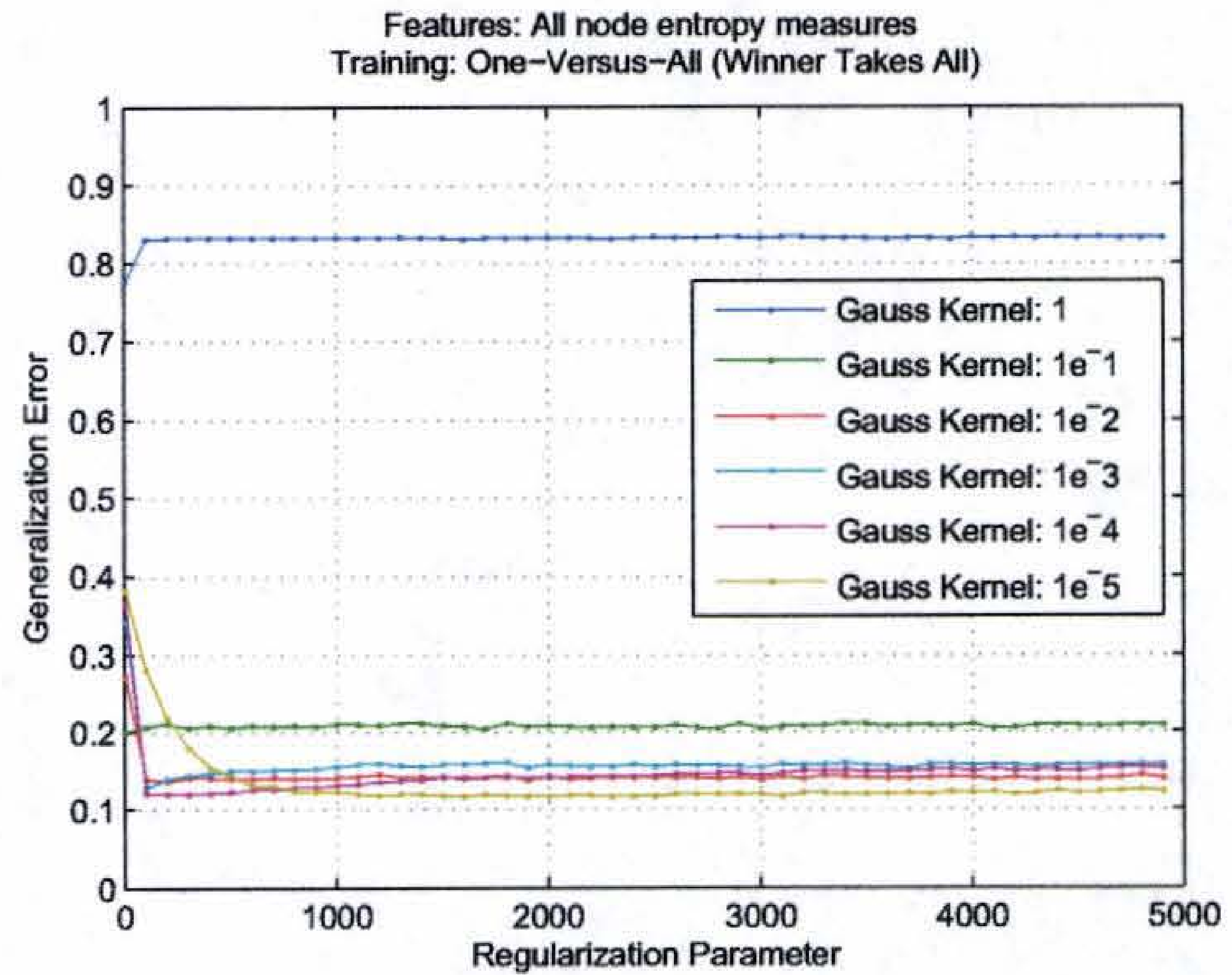

Figure 5.12: Sallen Key Bandpass FDI Generalization Error for One-verse-All Training with All Nodes for Features 
A comparison of the two best generalization error curves for each training method is displayed in Figure 5.13.

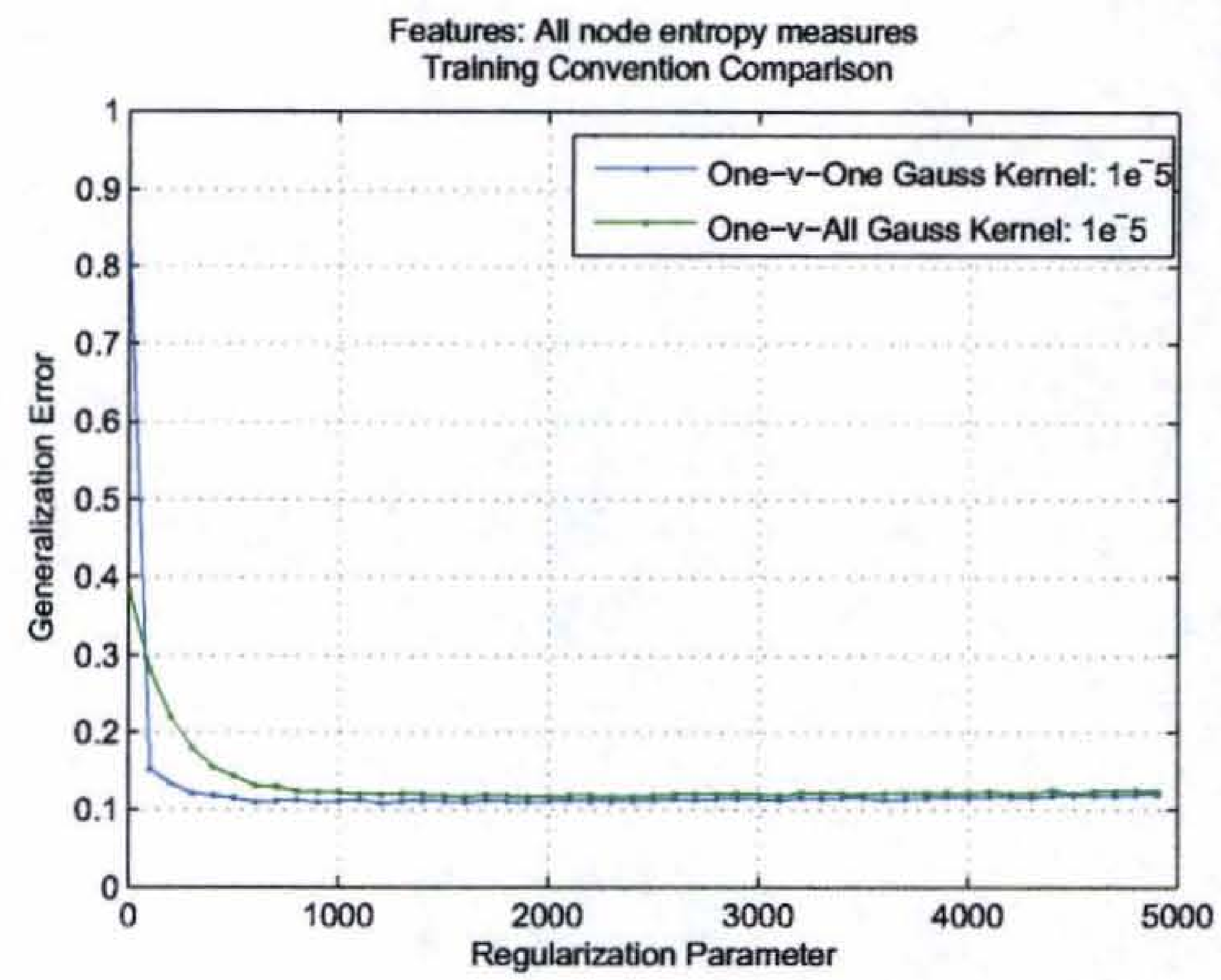

Figure 5.13: Sallen-Key bandpass filter FDI Generalization Error. Best Curve Comparison of two Training Conventions with all node entropy measures as features

Examination of Figure 5.16 reveals that the One-verses-One multi-class extension strategy slightly output performs the One-verses-All extension. A detailed comparison of the best performance from each of the multi-class extensions is given in Table 5.8.

\section{Features: Best basis node entropy measures}

This section presents the results of the FDI method which uses features constructed from the entropy measures based on the best basis algorithm described in Chapter 3. The results are broken down by training type (One-verses-One, One-verses-All). Figure 5.14 displays the performance of the One-verses-One multi-class extension for SVMs. The curves presented in Fig. 5.14 represent a series of fixed values for the Gaussian kernel parameter allowing the regularization parameter to vary. Examination of Figure 5.14 shows that good generalization performance for Pairwise SVM multi-class extension is achieved 
Table 5.8: Best Performance Breakdown: All Node Packets for the Sallen Key Bandpass Filter

\begin{tabular}{|c|c|c|}
\hline Fault Classes & $\begin{array}{c}\text { One-Versus-One } \\
\text { Error (\%) } \\
(C, \gamma)=\left(1200,1 * 10^{-5}\right)\end{array}$ & $\begin{array}{c}\text { One-Versus-All } \\
\text { Error (\%) } \\
(C, \gamma)=\left(2300,1 * 10^{-5}\right)\end{array}$ \\
\hline No Fault & 0.72 & 0.99 \\
\hline Faulty $R_{1}$ & 7.07 & 7.17 \\
\hline Faulty $R_{2}$ & 0.13 & 0.21 \\
\hline Faulty $R_{3}$ & 1.51 & 1.47 \\
\hline Faulty $C_{1}$ & 0.57 & 0.67 \\
\hline Faulty $C_{2}$ & 0.88 & 0.91 \\
\hline Total Error $(\%)$ & 10.90 & 11.44 \\
\hline
\end{tabular}

when the Gaussian kernel parameter is $1 * 10^{-4}$ and the regularization value 400 . Using these parameters a minimum generalization error of $10.6 \%$ is achieved.

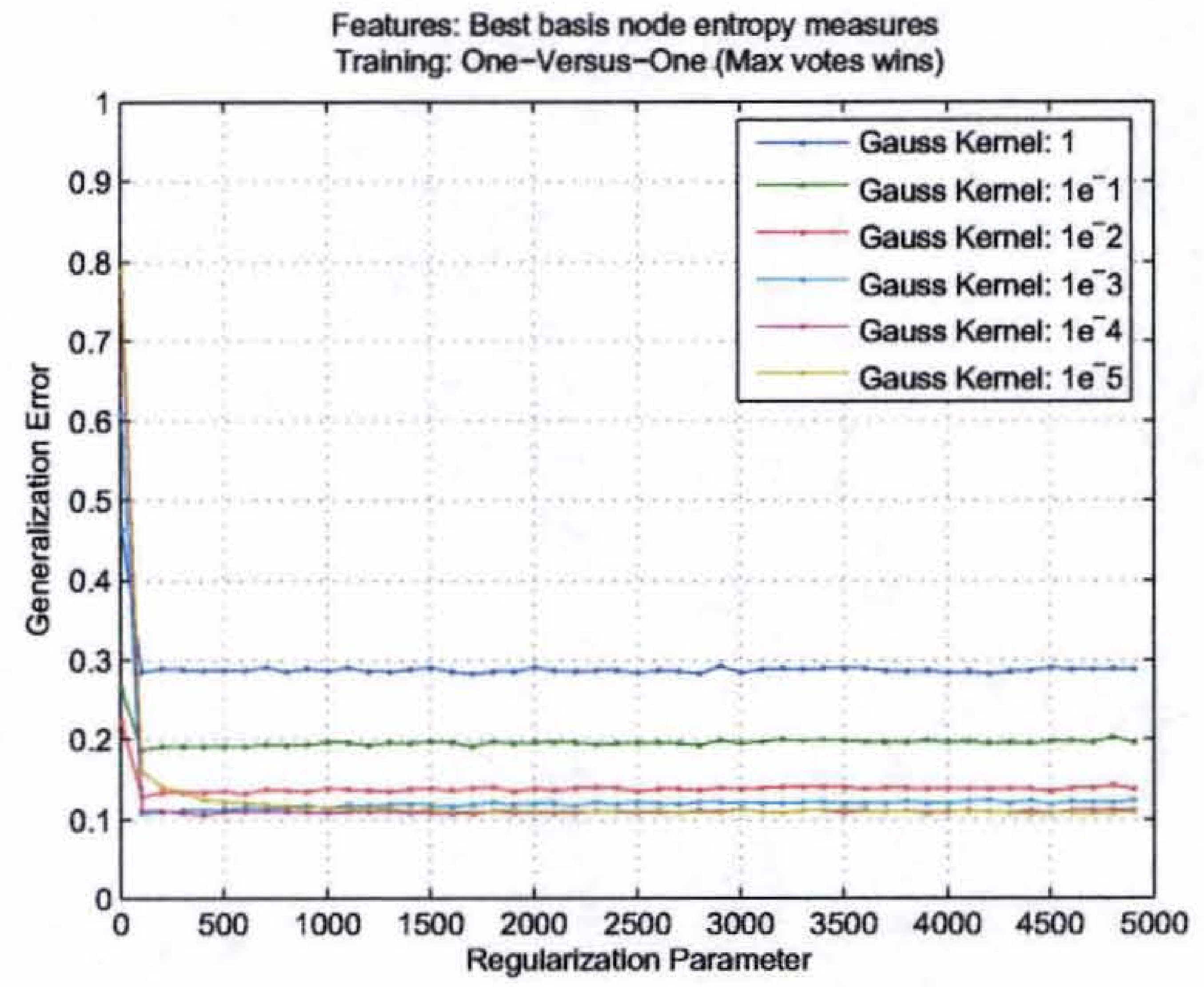

Figure 5.14: Sallen Key Bandpass FDI Generalization Error for One-verse-One Training with Best Basis Nodes for Features 
Figure 5.15 displays the performance of the One-verses-All multi-class extension for SVMs. The curves shown in Fig. 5.15 reflect the generalization performance for the various fixed Gaussian kernel parameters as the regularization parameter is varied. The best generalization performance of $11.0 \%$ is achieved when the Gaussian kernel parameter is $1 * 10^{-5}$ with the regularization parameter value of 4700 .

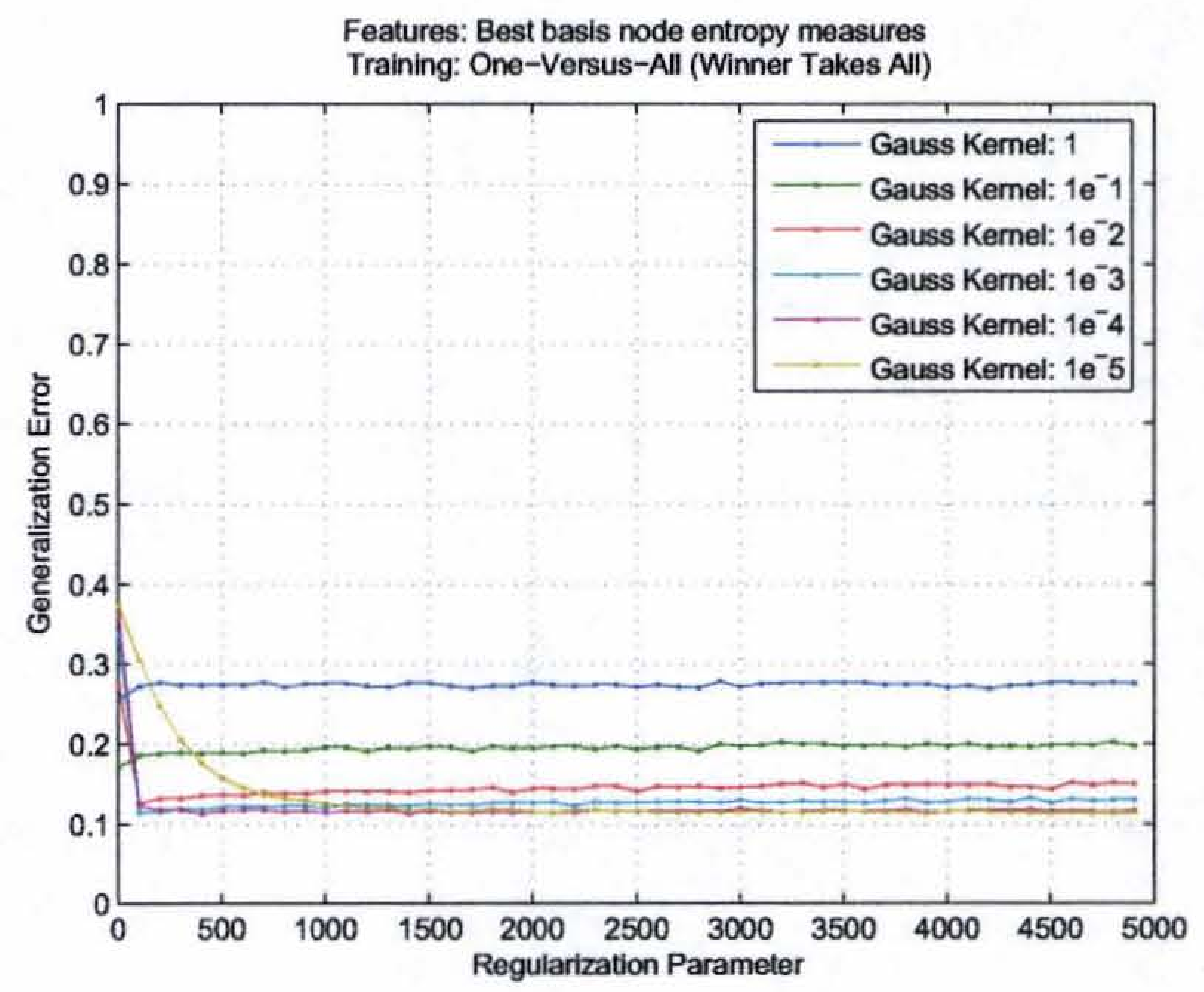

Figure 5.15: Sallen Key Bandpass FDI Generalization Error for One-verse-All Training with Best Basis Nodes for Features

A comparison of the two best generalization error curves for each training method is displayed in Figure 5.16. A detailed comparison of the best performance from each of the multi-class extensions is given in Table 5.9.

Table 5.10 summarizes the best performances from each of the feature representation methods. It can be seen that the fault diagnosis machine based on features from the best basis algorithm performs only slightly better than one which is trained on all node entropy measures. Similarly, there is no real difference in the performance of the two training techniques. 


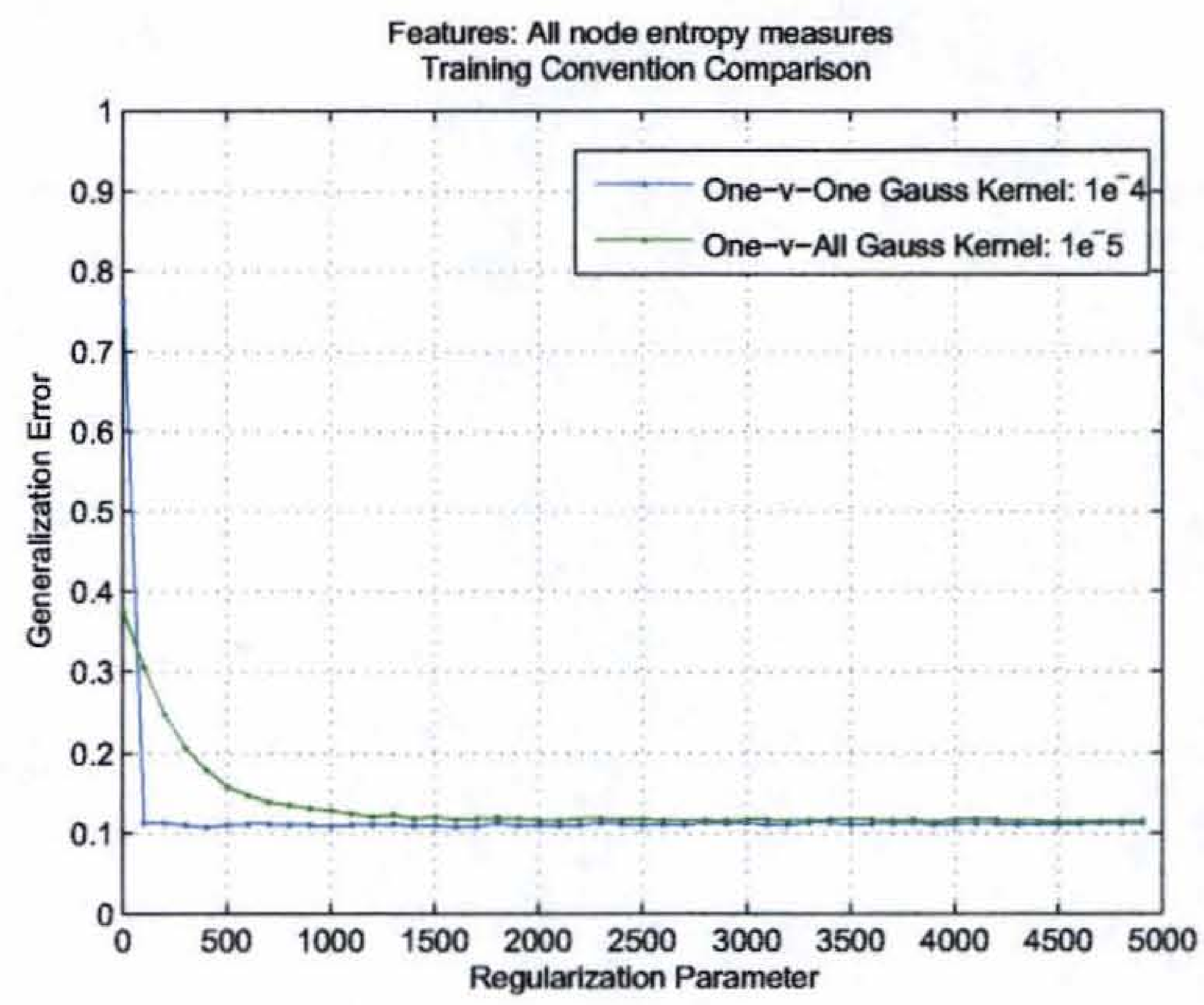

Figure 5.16: Sallen-Key bandpass filter FDI Generalization Error. Best Curve Comparison of two Training Conventions with all node entropy measures as features

Table 5.9: Best Performance Breakdown: Best Tree Node Packets for the Sallen Key Bandpass Filter

\begin{tabular}{|c|c|c|}
\hline Fault Classes & $\begin{array}{c}\text { One-Versus-One } \\
\text { Error (\%) } \\
(C, \gamma)=\left(400,1 * 10^{-4}\right)\end{array}$ & $\begin{array}{c}\text { One-Versus-All } \\
\text { Error (\%) } \\
(C, \gamma)=\left(4700,1 * 10^{-5}\right)\end{array}$ \\
\hline No Fault & 1.22 & 1.24 \\
\hline Faulty $R_{1}$ & 6.55 & 6.64 \\
\hline Faulty $R_{2}$ & 0.04 & 0.06 \\
\hline Faulty $R_{3}$ & 1.28 & 1.50 \\
\hline Faulty $C_{1}$ & 0.50 & 0.55 \\
\hline Faulty $C_{2}$ & 1.05 & 1.04 \\
\hline Total Error $(\%)$ & 10.66 & 11.07 \\
\hline
\end{tabular}


Table 5.10: Comparison of the Two Feature Representation Methods Best Performance for the Sallen Key Bandpass Filter

\begin{tabular}{c|cc|cc} 
& \multicolumn{2}{|c|}{ One-Versus-One } & \multicolumn{2}{c}{ One-Versus-All } \\
& $(C, \gamma)$ & Error $(\%)$ & $(C, \gamma)$ & Error (\%) \\
\hline $\begin{array}{c}\text { All Node Packets used } \\
\text { for Feature Representation }\end{array}$ & $\left(1 * 10^{-5}, 1200\right)$ & 10.90 & $\left(1 * 10^{-5}, 2300\right)$ & 11.44 \\
\hline $\begin{array}{c}\text { Best Basis Node Packets used } \\
\text { for Feature Representation }\end{array}$ & $\left(1 * 10^{-4}, 400\right)$ & 10.66 & $\left(1 * 10^{-5}, 4700\right)$ & 11.07 \\
\hline
\end{tabular}

\section{FDI performance under variable noise}

This section presents the results of the FDI scheme when the noise induced at $V_{o}$ is varied. The two different methods of feature representation are examined for each type of multi-class extension method. The respective FDI schemes are constructed based on the parameter values found in Table 5.10. With a fixed fault diagnosis machine the noise condition seen at $V_{o}$ is varied and the performance of each FDI method is seen displayed in Figure 5.17. Figure 5.17 shows that under noisy conditions SNR:-30dB - OdB the two different feature extraction methods perform about the same. However, when the SNR: is greater than $0 \mathrm{~dB}$ the fault diagnosis machine based on features from the best basis algorithm out performs one that is trained on all node entropy measures. This is most likely due to the fact that the best basis algorithm keeps only those nodes which significantly contribute to the representation of a signal. In effect the best basis algorithm is filtering out the unnecessary noise to some degree. 

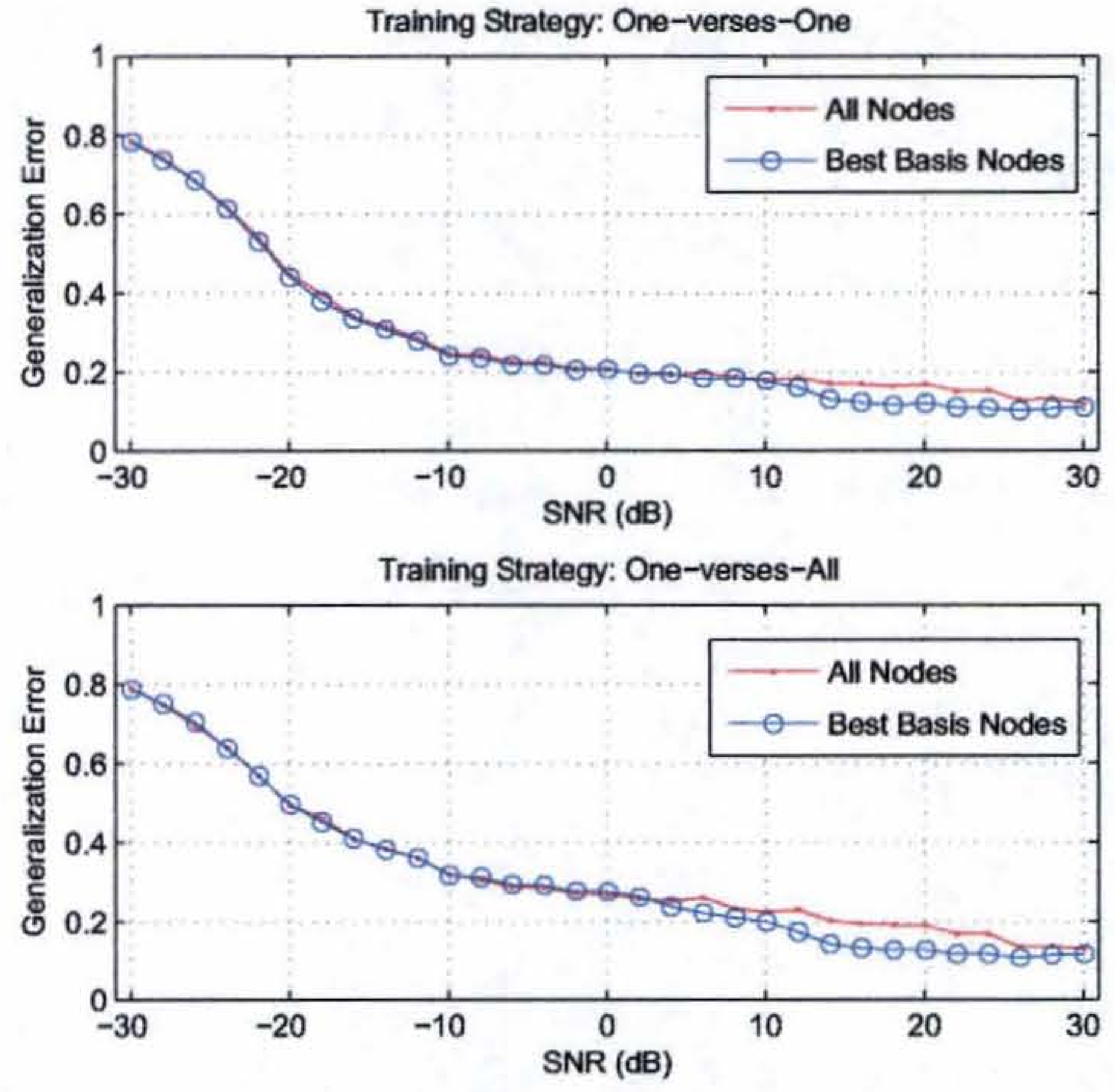

Figure 5.17: Effect of noise on FDI scheme for the Sallen-Key bandpass filter 


\section{Chapter 6}

\section{Conclusion}

\subsection{Discussions}

This thesis presented an effective data driven fault detection and identification scheme which was based on support vector machines and wavelet packet analysis. Support vector machines were considered as the diagnosis machine because of their effective use in pattern recognition problems. Also, because SVMs embed the problem of capacity control (overfitting) within the training algorithm and determination of support vectors. This avoids the curse of dimensionality that many of the feed forward neural networks suffer from. In fact SVMs are able to be a more generalized classifier because there is no need to include domain knowledge in their implementation. A preprocessing phase consisting of a wavelet packet transform and a entropy measure defined over the wavelet packet coefficients was used to provide the support vector machines with appropriate feature vectors.

Wavelet packet analysis was used as an effective means to draw out characteristic features which best defined each of the fault classes. The time signals could be used without any preprocessing for fault diagnosis but to do so usually results in poor performance of the diagnosis machine. This thesis presented a method which took raw outputs from a system and preprocessed them such that suitable feature vectors were used in the training of a diagnosis machine. In particular, two types of feature representation were studied. The first formed a feature vector for which the entropy metric was computed on all wavelet packet nodes in the decomposition tree. The second feature representation only computed 
the entropy measure on those nodes of the decomposition tree which made up the best basis. All other entropy measures were set to zero.

The proposed FDI scheme was applied to two different systems to demonstrate its robustness in application across various systems. In the attitude control system the FDI technique was applied to detect and isolate errors arising in the DC motor of the system. The proposed FDI scheme was able to accurately detect and identify faults arising from the armature inductance and resistance of the DC motor. To extend the FDI scheme to a more complicated system we investigated its use in detecting errors in a Sallen-Key bandpass filter. In particular, the FDI scheme was able to accurately detect and isolate errors within the components which made up the given filter. The proposed system was also able to perform relatively well even under noisy output measurements.

\subsection{Future Work}

This thesis only focused on the detection and isolation of occurring faults for a given system through SVMs and wavelet packet analysis. For future work it would be necessary to predict remaining life of a component once it has been isolated as the cause a detected fault. For instance, it is possible for a system to operate even though one of its components or subsystems has drifted beyond the designed limits. For this reason the system has not experienced a complete failure because of the component's deviation. However, it would only be a matter of time before the component completely fails causing a catastrophic failure. Therefore, it would be beneficial if a FDI system were to not simply able isolate faults but to also estimate the remaining life until critical failure. This type of fault analysis falls under the heading of pyrognostics. This could be accomplished by a variety of methods one being the incorporation of SVMs for regression problems. Also, this investigation only considered faults occurring one at a time. It would be beneficial to examine situations where faults occurred in combination with one another. 


\section{Bibliography}

[1] F. Aminian and M. Aminian. Neural-network based analog-circuit fault diagnosis using wavelet transform as preprocessor. IEEE Transactions on Circuits and Systems II, 47:151-156, February 2000.

[2] G. Bachman, L. Narici, and E. Beckenstein. Fourier and Wavelet Analysis. SpringerVerlag, New York, 2000.

[3] B.cholkopf and A.J. Smola. Learning with Kernels. The MIT Press, Cambridge, MA, 2002.

[4] D.P. Bertsekas. Nonlinear Programming. Athenas Scientific, Belmont, MA, 1995.

[5] C. M. Bishop. Neural Networks for Pattern Recognition. Oxford University Press, 1995.

[6] Christian Blatter. Wavelets A Primer. A. K. Peters Ltd., 1998.

[7] C.J.C. Burges. A tutorial on support vector machines for pattern recognition. Data Mining and Knowledge Discovery, 2:121-167, 1998.

[8] M. Catelani and A. Fort. Soft fault detection and isolation in analog circuits: Some results and a comparison between a fuzzy approach and radial basis function networks. IEEE Transactions on Instrumentaion and Measurement, 51:196-202, April 2002.

[9] M. Cody. The wavelet packet transform extending the wavelet transform. Dr. Dobb's Journal, pages 44-54, 1994.

[10] R.R. Coifman and M.V. Wickerhauser. Entropy-based algorithms for best basis selection. IEEE Transactions on Information Theory, 38:713-718, March 1992. 
[11] R.A. Gopinath C.S. Burrus and H. Guo. Introduction to Wavelets and Wavelet Transforms A Primer. Prentice-Hall, New Jersey, 1998.

[12] I. Daubechies. Ten Lectures on Wavelets. Soecity for Industrial and Applied Mathematics, Philadelphia, 1992.

[13] Laurene Fausett. Fundamentals of Neural Networks. Prentice-Hall, 1994.

[14] R. J. Ferlez and D. C. Lang. Gear-tooth fault detection and tracking using the wavelet transform. In Proceedings of 52nd Meeting Society for Machinery Failure Prevention, pages $451-460,1998$.

[15] H. Xing H. Zhonghui, C. Yunze and X. Xiaoming. Fusion of multi-class support vector machines for fault diagnosis. In Proceedings of the 2005 American Control Conference, volume 3, pages 1941-1945, June 2005.

[16] Mohamad H. Hassoun. Fundamentals of Artificial Neural Networks. MIT Press, 1995.

[17] Simon Haykin. Neural Networks a Comprehensive Foundation. Prentice-Hall, New Jersey, 1999.

[18] R. Hetch-Nielsen. Neurocomputing. Addison-Wesley, 1990.

[19] B. B. Hubbard. The world according to wavelets. A. K. Peters Ltd., 1996.

[20] M.V. Casique J. Dehmeshki, J. Chen and M. Karakoy. Classification of lung data by sampling and support vector machine. In Proceedings of the 2004 Engineering in Medicine and Biology Society, volume 2, pages 3194-3197, 2004.

[21] B.C. Kuo. Automatic Control Systems. Pretince Hall, New Jersey, 1991.

[22] B. Liu and S. F. Ling. Machinery diagnosis based on wavelet packets. Journal of Vibration and Control, 3:5-17, January 1997.

[23] S. Mallat. A Wavelet Tour of Signal Processing. Academic Press, San Diego, 1998. 
[24] P. Frank R. Patton and R. Clark. Fault Diagnosis in Dynamic Systems Theory and Application. Prentice-Hall, New Jersey, 1989.

[25] R. Spina and S. Upadhyaya. Linear circuit fault diagnosis using neuromorphic analyzers. IEEE Transactions on Circuits and Systems II, 44:188-196, March 1997.

[26] V. Vapnik. Statistical Learning Theory. Wiley, New York, 1998.

[27] James S. Walker. A Primer On Wavelets and their Scientific Applicaions. Chapman and Hall CRC, 1999.

[28] J. Liu X. Liu, H. Zhang and J. Yang. Fault detection and diagnosis of permanentmagnet dc motor based on parameter estimation and neural network. IEEE Transactions on Industrial Electronics, 47:1021-1030, October 2000.

[29] M. Binonwangan X. Wang and L. S. Vassilis. Vehicle health monitoring system using multiple-model adaptive estimation. In Proceedings of 11 th Mediterranean Conference on Control and Automation, 2003.

[30] G. Yen and K. Lin. Wavelet packet feature extraction for vibration monitoring. IEEE Transactions on Industrial Electronics, 47:650-667, June 2000. 